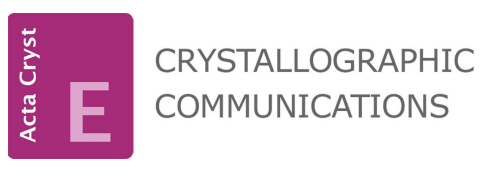

ISSN 2056-9890

Received 8 May 2020

Accepted 18 May 2020

Edited by S. Parkin, University of Kentucky, USA

Keywords: anticancer; azelaic acid; co-crystal; crystal structure; gefitinib; hydrogen bond; $\pi-\pi$ interactions.

CCDC reference: 2002536

Supporting information: this article has supporting information at journals.iucr.org/e

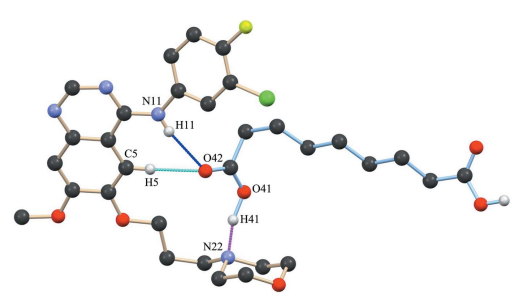

\section{Crystal structure of a 1:1 co-crystal of the anti- cancer drug gefitinib with azelaic acid}

\author{
Christy P. George, ${ }^{a}$ Ekta Sangtani ${ }^{\mathrm{a}}$ and Rajesh G. Gonnade ${ }^{\mathrm{a}, \mathrm{b} *}$ \\ aPhysical and Materials Chemistry Division, CSIR-National Chemical Laboratory, Pune-411008, India, and ${ }^{\mathbf{b}}$ Academy of \\ Scientific and Innovative Research (AcSIR), Ghaziabad, Uttar Pradesh-201002, India. *Correspondence e-mail: \\ rg.gonnade@ncl.res.in
}

In the title co-crystal, $\mathrm{C}_{22} \mathrm{H}_{24} \mathrm{ClFN}_{4} \mathrm{O}_{3} \cdot \mathrm{C}_{9} \mathrm{H}_{16} \mathrm{O}_{4}$, gefitinib (GTB; systematic name: quinazolin-4-amine) co-crystallizes with azelaic acid (AA; systematic name: nonanedioic acid). The co-crystal has the monoclinic $P 2_{1} / n$ centrosymmetric space group, containing one molecule each of GTB and AA in the asymmetric unit. A structure overlay of the GTB molecule in the co-crystal with that of its most stable polymorph revealed a significant difference in the conformation of the morpholine moiety. The significant deviation in the conformation of one of the acidic groups of azelaic acid from its usual linear chain structure could be due to the encapsulation of one acidic group in the pocket formed between the two pincers of GTB namely, the morpholine and phenyl moieties. Both GTB and AA molecules form $\mathrm{N}-\mathrm{H} \cdots \mathrm{O}, \mathrm{O}-\mathrm{H} \cdots \mathrm{N}, \mathrm{C}-$ $\mathrm{H}$. . O hydrogen bonds with $\mathrm{C}-\mathrm{H}$. . F close contacts along with off-stacked aromatic $\pi-\pi$ interactions between the GTB molecules.

\section{Chemical context}

Gefitinib (GTB, Iressa) is an orally administered chemotherapy treatment drug that inhibits tyrosine kinase (an enzyme that transports phosphates from ATP to the tyrosine residue of a protein) (Kobayashi \& Hagiwara, 2013) for nonsmall-cell lung cancer (NSCLC), pancreatic cancer, breast cancer and several other types of cancer. Two polymorphs of GTB have been reported from our group previously, both of which crystallized in the triclinic $P \overline{1}$ space group (Thorat et al., 2014). The drug-drug co-crystal of GTB with furosemide has also been published (Thorat et al., 2015). Some of the major side effects of GTB include rash, acne and dry skin. To overcome these after effects, there is a need for combination drug therapy. In this regard, we chose azelaic acid (AA), which is used for treating mild to moderate acne, both comedonal acne and inflammatory acne (Fitton \& Goa, 1991). Furthermore, GTB is also known to form co-crystals with aliphatic dicarboxylic acids through $\mathrm{N}-\mathrm{H} \cdots \mathrm{O}$ and $\mathrm{O}-\mathrm{H} \cdots \mathrm{N}$ hydrogen bonds (Gonnade, 2015). AA is an aliphatic dicarboxylic acid (heptane-dicarboxylic acid), having seven $\mathrm{CH}_{2}$ groups in the alkyl chain. Two polymorphs of AA have been reported earlier, the $\alpha$ form is monoclinic, $P 2_{1} / c$ (Caspari, 1928; Housty $\&$ Hospital, 1967) and the $\beta$ form crystallizes in the monoclinic $C 2 / c$ space group (Housty \& Hospital, 1967). Both GTB and AA are non-volatile solids at room temperature and their respective melting points are in the ranges 192-195 K and 378-381 K. 
<smiles>COc1cc2ncnc(Nc3ccc(F)c(Cl)c3)c2cc1OCCCN1CCOCC1</smiles>

\section{Structural commentary}

The title compound GTB-AA (1:1) crystallizes in the monoclinic $P 2_{1} / n$ centrosymmetric space group containing one molecule of each in the asymmetric unit (Fig. 1, Table 1) (CCDC reference No. 2002536). The halophenyl ring of GTB and the alkyl $\left(-\mathrm{CH}_{2}-\right)$ chain of AA exhibit positional disorder over two conformations, due to the free rotation around the $\mathrm{N}-\mathrm{C}$ and $\mathrm{C}-\mathrm{C}$ single bonds, respectively (Fig. $2 a$ and $2 b$ ). A structure overlay of the GTB molecule based on a fit of the quinazoline groups in the co-crystal structure with that of its stable polymorph [the crystal structure of the stable polymorph of GTB was retrieved from the Cambridge Structural Database (Groom et al., 2016), refcode: FARRUM02; Thorat et al., 2014] revealed a considerable difference in the orientation of the morpholine moiety [torsion angles, C19-C20$\mathrm{C} 21-\mathrm{N} 22=54.0(2)^{\circ}$ for GTB in the co-crystal while the corresponding torsion angle in the stable polymorph of GTB is

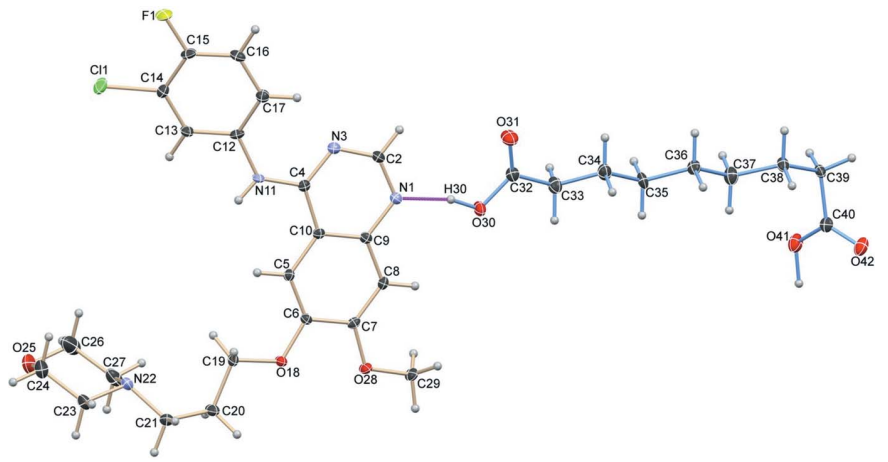

Figure 1

The asymmetric unit of the title compound, showing the atom labelling, $50 \%$ probability displacement ellipsoids for non- $\mathrm{H}$ atoms and hydrogen bonding with a dotted magenta line. $\mathrm{H}$ atoms are shown as small spheres of arbitrary radii.

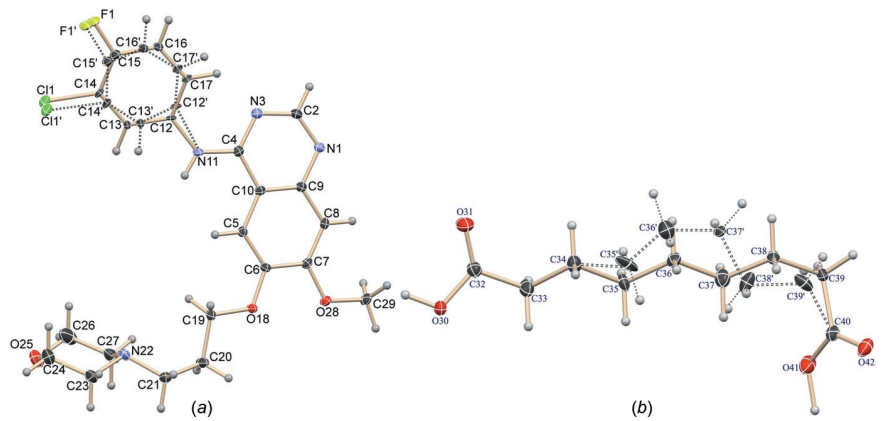

Figure 2

Crystal structures of GTB $(a)$ and AA $(b)$ in the co-crystal showing positional disorder of the halophenyl ring and alkyl chain, respectively.
Table 1

Hydrogen-bond geometry $\left(\AA{ }^{\circ}\right)$.

\begin{tabular}{lllll}
\hline$D-\mathrm{H} \cdots A$ & $D-\mathrm{H}$ & $\mathrm{H} \cdots A$ & $D \cdots A$ & $D-\mathrm{H} \cdots A$ \\
\hline $\mathrm{N} 11-\mathrm{H} 11 \cdots \mathrm{O} 42^{\mathrm{i}}$ & $0.84(2)$ & $2.20(2)$ & $3.025(2)$ & $169.6(18)$ \\
$\mathrm{O} 41-\mathrm{H} 41 \cdots \mathrm{N} 22^{\text {ii }}$ & 1.01 & 1.78 & $2.6566(19)$ & 144 \\
$\mathrm{O} 30-\mathrm{H} 30 \cdots \mathrm{N} 1$ & $0.99(3)$ & $1.65(3)$ & $2.6135(19)$ & $166(2)$ \\
$\mathrm{C} 5-\mathrm{H} 5 \cdots \mathrm{O} 42^{\mathrm{i}}$ & 0.95 & 2.25 & $3.194(2)$ & 170 \\
$\mathrm{C} 2-\mathrm{H} 2 \cdots 1^{\text {iii }}$ & 0.95 & 2.15 & $3.07(3)$ & 163 \\
$\mathrm{C} 2-\mathrm{H} 2 \cdots 1^{\text {iii }}$ & 0.95 & 2.32 & $3.253(3)$ & 166 \\
$\mathrm{C} 29-\mathrm{H} 29 B \cdots \mathrm{O} 18^{\text {iv }}$ & 0.98 & 2.65 & $3.6101(19)$ & 167 \\
$\mathrm{C} 23-\mathrm{H} 23 B \cdots \mathrm{O} 25^{\mathrm{v}}$ & 0.99 & 2.57 & $3.220(2)$ & 123 \\
$\mathrm{C} 27-\mathrm{H} 27 B \cdots \mathrm{F} 1^{\text {vi }}$ & 0.99 & 2.71 & $3.68(4)$ & 166 \\
$\mathrm{C} 39-\mathrm{H} 39 A \cdots \mathrm{O} 30^{\text {vii }}$ & 0.99 & 2.50 & $3.255(4)$ & 133 \\
$\mathrm{C} 21-\mathrm{H} 21 B \cdots \mathrm{O} 31^{\text {viii }}$ & 0.99 & 2.29 & $3.234(2)$ & 160 \\
$\mathrm{C} 13-\mathrm{H} 13 \cdots \mathrm{O} 30^{\text {ix }}$ & 0.95 & 2.39 & $3.139(3)$ & 135 \\
$\mathrm{C} 13^{\prime}-\mathrm{H} 13^{\prime} \cdots \mathrm{O} 30^{\text {ix }}$ & 0.95 & 2.53 & $3.268(3)$ & 135 \\
$C g 2 \cdots C g 2^{\text {viii }}$ & & & $3.5358(11)$ & $0(1)$ \\
$C g 2 \cdots C g 3^{\text {viii }}$ & & & $3.7909(11)$ & $1(1)$ \\
$C g 2 \cdots C g 3^{\text {ix }}$ & & & $3.7530(11)$ & $1(1)$ \\
$C g 3 \cdots C g 3^{\text {viii }}$ & & & $3.7934(11)$ & $0(1)$
\end{tabular}

Symmetry codes: (i) $\quad x-\frac{1}{2},-y+\frac{3}{2}, z-\frac{1}{2}$; (ii) $x+\frac{1}{2},-y+\frac{3}{2}, z+\frac{1}{2}$; $-x,-y+2,-z+1$; (iv) $-x+2,-y+1,-z+1$; (v) $-x+\frac{3}{2}, y-\frac{1}{2},-z+\frac{1}{2}$; (vi) $x+1, y, z ; \quad$ (vii) $\quad-x+\frac{3}{2}, y+\frac{1}{2},-z+\frac{3}{2} ; \quad$ (viii) $\quad-x+1,-y+1,-z+1$; $\quad$ (ix) $-x+1,-y+2,-z+1$.

$\left.74.3(2)^{\circ}\right]$ because of the conformationally flexible $-\mathrm{CH}_{2}-$ spacer (Fig. 3). Whereas the conformation of the phenyl group showed a slight difference with a dihedral angle of $14.1(2)^{\circ}$ (the angular difference between the planes of halophenyl ring of both structures). The quinazoline, morpholine and phenyl moieties of GTB have acquired a roughly planar geometry in the co-crystal [torsion angle $\mathrm{C} 12-\mathrm{C} 5-\mathrm{C} 19-\mathrm{N} 22=14.4(2)^{\circ}$, only the $\mathrm{N}$ atom of morpholine is considered and not the full fragment], whereas in the stable polymorph of GTB, the morpholine moiety deviates significantly from the plane [the corresponding torsion angle is $\left.-75.7(2)^{\circ}\right]$. The approximate planarity of the phenyl, quinazoline and morpholine (only $\mathrm{N}$ atom considered) moieties of GTB in the co-crystal seems to be due to the engagement of these groups with one of the acid groups of AA via $\mathrm{N}-\mathrm{H} \cdots \mathrm{O}$ and $\mathrm{O}-\mathrm{H} \cdots \mathrm{N}$ hydrogen bonds. The conformation of this acid group of AA shows a consid-

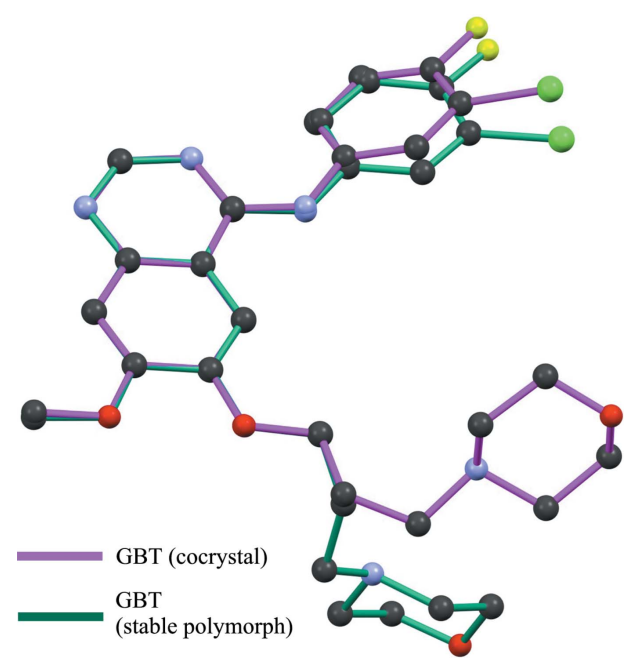

Figure 3

Structure overlay of GTB molecule in the co-crystal (magenta) and its stable polymorph (green). 


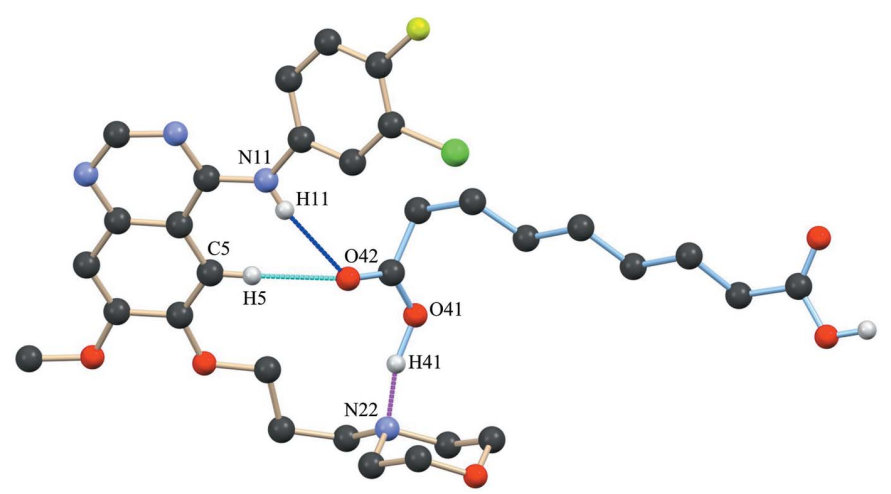

Figure 4

The 'molecular clip'-like geometry of GTB that accommodates a carboxyl group of AA. The molecules interact through $\mathrm{N}-\mathrm{H} \cdots \mathrm{O}, \mathrm{O}-\mathrm{H} \cdots \mathrm{N}$ and $\mathrm{C}-\mathrm{H} \cdots \mathrm{O}$ hydrogen bonds.

erable departure from its usual linear chain structure due to an acquired bend at the 7th carbon atom (C39) [torsional difference $105.15(19)^{\circ}$ from the other end of the acid group, torsion angles, $\mathrm{C} 32-\mathrm{C} 33-\mathrm{C} 34-\mathrm{C} 35=-174.15(19)^{\circ}$ and $\left.\mathrm{C} 37-\mathrm{C} 38-\mathrm{C} 39-\mathrm{C} 40=-69.0(3)^{\circ}\right]$. The conformational bend could be due to the inclusion of the acid moiety in the pocket formed between the morpholine and phenyl moieties (which have a molecular clip-like geometry) of GTB and the subsequent involvement of the carbonyl and hydroxyl groups of the included acid moiety in the formation of the $\mathrm{N}-\mathrm{H} \cdots \mathrm{O}$ and $\mathrm{O}-\mathrm{H} \cdots \mathrm{N}$ hydrogen bonds with the distantly located amine $\mathrm{N}-\mathrm{H}$ and the $\mathrm{N}$ atom of the morpholine moiety, respectively (Fig. 4). The other acid group of AA forms an $\mathrm{O}-\mathrm{H} \cdots \mathrm{N}$ hydrogen bond with the $\mathrm{N}$ atom of the quinazoline moiety.

\section{Supramolecular features}

The closely associated molecules of GTB and AA (through an $\mathrm{O} 30-\mathrm{H} 30 \cdots \mathrm{N} 1$ hydrogen bond) constitute a 'zero-dimensional' supramolecular motif wherein a carboxyl OH of AA donates its $\mathrm{H}$ atom to the quinazoline $\mathrm{N}$ atom (Fig. 1). Adjacent $n$-glide symmetry-related 'zero-dimensional' motifs are linked firmly along the $a c$ diagonal by strong $\mathrm{N}-\mathrm{H} \cdots \mathrm{O}$, $\mathrm{O}-\mathrm{H} \cdots \mathrm{N}$ and $\mathrm{C}-\mathrm{H} \cdots \mathrm{O}$ hydrogen bonds to generate a onedimensional linear chain structure (Fig. 5, Table 1). The cavity created by GTB as a result of its 'molecular clip'-like geometry

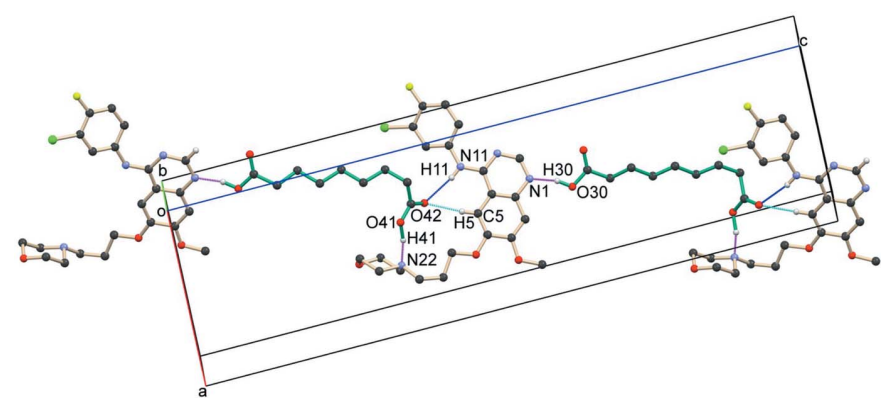

Figure 5

A one-dimensional chain formed by GTB and AA molecules along the $a c$ diagonal via $\mathrm{O}-\mathrm{H} \cdots \mathrm{N}, \mathrm{N}-\mathrm{H} \cdots \mathrm{O}$ and $\mathrm{C}-\mathrm{H} \cdots \mathrm{O}$ hydrogen bonds.

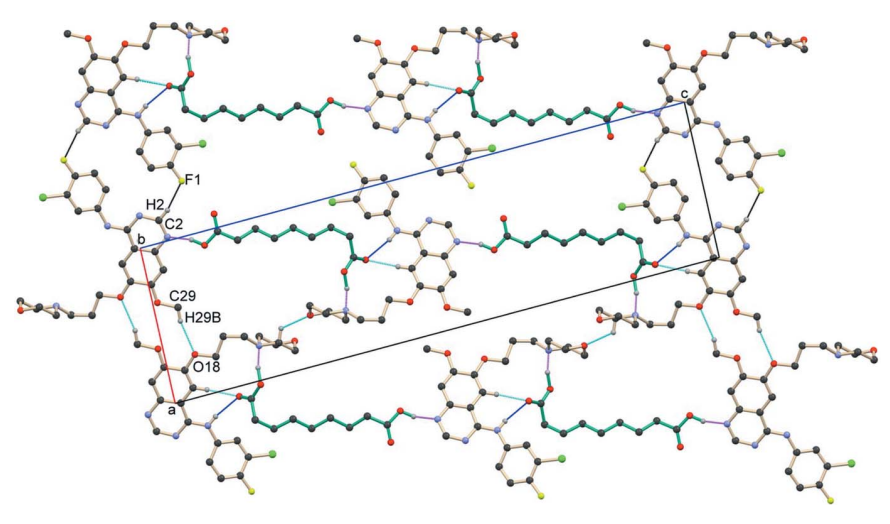

Figure 6

Two-dimensional layered assembly of GTB and AA along the ac diagonal. The neighbouring one-dimensional chains are stitched through $\mathrm{C}-\mathrm{H} \cdots \mathrm{F}$ and $\mathrm{C}-\mathrm{H} \cdots \mathrm{O}$ hydrogen bonds.

encapsulates the other carboxylic acid group of AA. In the cavity, the carboxyl oxygen (O42) accepts the $\mathrm{H}$ atoms from amine $\mathrm{N} 11-\mathrm{H} 11$ and $\mathrm{C} 5-\mathrm{H} 5$ to form $\mathrm{N} 11-\mathrm{H} 11 \cdots \mathrm{O} 42^{\mathrm{i}}$ and $\mathrm{C} 5-\mathrm{H} 5 \cdots \mathrm{O} 42^{\mathrm{i}}$ hydrogen bonds (symmetry operations are given in the footnote to Table 1). In turn, the carboxyl $\mathrm{OH}$ $(\mathrm{O} 41-\mathrm{H} 41)$ of AA donates its $\mathrm{H}$ atom to the morpholine $\mathrm{N} 22$ to make a $\mathrm{O} 41-\mathrm{H} 41 \cdots \mathrm{N} 22^{\mathrm{ii}}$ hydrogen bond. The neighbouring antiparallel chains are stitched centrosymmetrically through $\mathrm{C} 2-\mathrm{H} 2 \cdots \mathrm{F}^{\mathrm{iii}}$ contacts and $\mathrm{C} 29-\mathrm{H} 29 B \cdots \mathrm{O} 18^{\mathrm{iv}}$ hydrogen bonds to form a two-dimensional layered assembly in the $a c$ plane (Fig. 6). A view of the molecular packing down the $b$ axis reveals the stacking of the 2D layers by aromatic $\pi-$ $\pi$ interactions between centrosymmetrically related quinazoline rings [interplanar spacing, $3.396(13) \AA]\left(C g 2 \cdots C g 2^{\text {vii }}\right.$, $C g 2 \cdots C g 3^{\text {vii }}, C g 2 \cdots C g 3^{\text {viii }}$ and $C g 3 \cdots C g 3^{\text {vii }} ; C g 2$ is the centroid of the $\mathrm{N} 1 / \mathrm{C} 2 / \mathrm{N} 3 / \mathrm{C} 4 / \mathrm{C} 10 / \mathrm{C} 9$ ring and $C g 3$ is the centroid of the $\mathrm{C} 5-\mathrm{C} 10$ ring, Table 1). Molecules between the two layers are also connected by $\mathrm{C} 27-\mathrm{H} 27 B \cdots \mathrm{F}^{\mathrm{vi}}$ contacts and $\mathrm{C} 23-\mathrm{H} 23 B \cdots \mathrm{O} 25^{\mathrm{v}}, \quad \mathrm{C} 21-\mathrm{H} 21 B \cdots \mathrm{O} 31^{\mathrm{vii}}, \quad \mathrm{C} 13-$ $\mathrm{H} 13 \cdots \mathrm{O} 30^{\text {viii }}$ and $\mathrm{C} 39-\mathrm{H} 39 A \cdots \mathrm{O} 30^{\mathrm{ix}}$ hydrogen bonds to generate the three-dimensional packing (Fig. 7, Table 1).

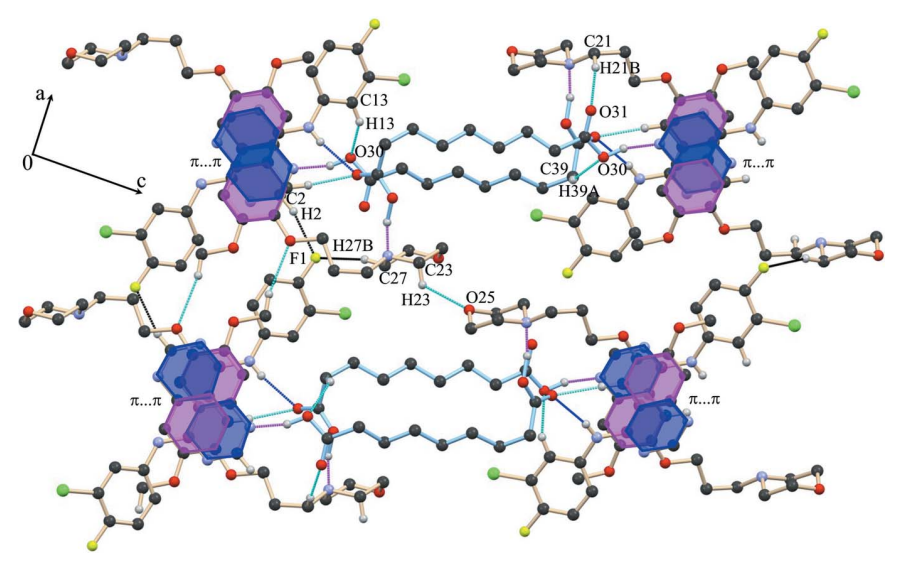

Figure 7

The view of the molecular packing along the $b$ axis showing the association of GTB molecules through aromatic $\pi-\pi$ interactions along with $\mathrm{C}-\mathrm{H} \cdots \mathrm{F}$ and $\mathrm{C}-\mathrm{H} \cdots \mathrm{O}$ interactions. 


\section{Database survey}

A search for the title co-crystal in the Cambridge Structural Database (CSD, Version 5.41, the update of March 2020; Groom et al., 2016) found no hits. However, searches for GTB and AA gave 8 and 35 hits, respectively. A search for the GTB molecule showed that the amine $\mathrm{N}-\mathrm{H}$ moiety is involved in $\mathrm{N}-\mathrm{H} \cdots \mathrm{O}$ hydrogen-bond formation either with the morpholine oxygen in both of its polymorphs (Thorat et al., 2014 ) or with the water oxygen (Gilday et al., 2005; Thorat et $a l ., 2015)$. For the AA search, 17 hits were found only for its two polymorphs (refcodes: AZELAC01-AZELAC17) wherein the AA molecules are found to be associated by the conventional dimeric $\mathrm{O}-\mathrm{H} \cdots \mathrm{O}$ hydrogen bonds (Caspari, 1928; Housty \& Hospital, 1967). The remaining hits were for either co-crystals with amides (Tothadi \& Phadkule, 2019; Thompson et al., 2011; Karki et al., 2009), pyridines (Braga et al., 2010; Martins et al., 2016; Krueger et al., 2017) or complexes with Ni (Zhao et al., 2012), Fe (Braga et al., 2006) or Ba (Grzesiak et al., 2012).

\section{Synthesis and crystallization}

Co-crystallization was carried out using equimolar amounts of commercial samples of GTB and AA by grinding combined with a slow evaporation method. The grinding experiment was performed manually using a mortar and pestle. The 1:1 stoichiometric molar ratio of GTB (45 mg, $0.1 \mathrm{mmol})$ and AA (19 mg, $0.1 \mathrm{mmol}$ ) was ground for about 15 minutes using dry (neat) grinding. The ground sample was dissolved in $n$-butanol and heated for $\sim 10$ minutes to ensure the complete dissolution of the sample. The solution was filtered into the crystallization flask to remove the impurity and undissolved compound, and the solution was allowed to evaporate at room temperature (298-300 K). Elongated needle-shaped colourless crystals were obtained after $1-2 \mathrm{~h}$. The melting point of the obtained co-crystal was 398-399 K.

\section{Refinement}

Crystal data, data collection and structure refinement details are summarized in Table 2. All $\mathrm{H}$ atoms (except for hydroxy and amine $\mathrm{H}$ atoms) were placed in geometrically idealized positions, with $\mathrm{C}-\mathrm{H}=0.95 \AA$ for phenyl $\mathrm{H}$ atoms, $\mathrm{C}-\mathrm{H}=$ $0.99 \AA$ for methylene $\mathrm{H}$ atoms and $\mathrm{C}-\mathrm{H}=0.98 \AA$ for methyl $\mathrm{H}$ atoms. They were constrained to ride on their parent atoms, with $U_{\text {iso }}(\mathrm{H})=1.2 U_{\text {eq }}(\mathrm{C})$ for phenyl and methylene, and $1.5 U_{\text {eq }}(\mathrm{C})$ for methyl groups. The O- $(\mathrm{O} 30)$ and $\mathrm{N}$-bound $\mathrm{H}$ atoms were located in difference-Fourier maps and refined isotropically. However, the O-bound $\mathrm{H}$ atom was placed in a geometrically idealized positions using HFIX 148 as the $\mathrm{O}-\mathrm{H}$ distance was longer when refined with its located position in the difference-Fourier map. It was constrained to ride on its parent atom $(\mathrm{O} 41)$, with $U_{\text {iso }}(\mathrm{H})=1.5 U_{\text {eq }}(\mathrm{O})$. The long $\mathrm{O}-\mathrm{H}$ distance could be due to its involvement in the strong $\mathrm{O}-$ $\mathrm{H} \cdots \mathrm{N}$ hydrogen-bond formation with $\mathrm{N} 22$. The difference
Table 2

Experimental details.

\begin{tabular}{|c|c|}
\hline \multicolumn{2}{|l|}{ Crystal data } \\
\hline Chemical formula & $\mathrm{C}_{22} \mathrm{H}_{24} \mathrm{ClFN}_{4} \mathrm{O}_{3} \cdot \mathrm{C}_{9} \mathrm{H}_{16} \mathrm{O}_{4}$ \\
\hline$M_{\mathrm{r}}$ & 635.12 \\
\hline Crystal system, space group & Monoclinic, $P 2_{1} / n$ \\
\hline Temperature (K) & 100 \\
\hline$a, b, c(\AA)$ & $\begin{array}{l}10.7716(10), 7.4153(13) \\
\quad 38.175(7)\end{array}$ \\
\hline$\beta\left({ }^{\circ}\right)$ & $92.311(5)$ \\
\hline$V\left(\AA^{3}\right)$ & $3046.7(8)$ \\
\hline$Z$ & 4 \\
\hline Radiation type & Mo $K \alpha$ \\
\hline$\mu\left(\mathrm{mm}^{-1}\right)$ & 0.19 \\
\hline Crystal size (mm) & $0.28 \times 0.19 \times 0.04$ \\
\hline \multicolumn{2}{|l|}{ Data collection } \\
\hline Diffractometer & $\begin{array}{c}\text { Bruker D8 VENTURE Kappa } \\
\text { Duo PHOTON II CPAD }\end{array}$ \\
\hline Absorption correction & $\begin{array}{l}\text { Multi-scan (SADABS; Bruker, } \\
\text { 2016) }\end{array}$ \\
\hline$T_{\min }, T_{\max }$ & $0.950,0.993$ \\
\hline $\begin{array}{l}\text { No. of measured, independent and } \\
\text { observed }[I>2 \sigma(I)] \text { reflections }\end{array}$ & $92337,7338,5413$ \\
\hline$R_{\text {int }}$ & 0.162 \\
\hline$(\sin \theta / \lambda)_{\max }\left(\AA^{-1}\right)$ & 0.661 \\
\hline \multicolumn{2}{|l|}{ Refinement } \\
\hline$R\left[F^{2}>2 \sigma\left(F^{2}\right)\right], w R\left(F^{2}\right), S$ & $0.045,0.110,1.02$ \\
\hline No. of reflections & 7338 \\
\hline No. of parameters & 473 \\
\hline No. of restraints & 126 \\
\hline $\mathrm{H}$-atom treatment & $\begin{array}{l}\mathrm{H} \text { atoms treated by a mixture of } \\
\text { independent and constrained } \\
\text { refinement }\end{array}$ \\
\hline$\Delta \rho_{\max }, \Delta \rho_{\min }\left(\mathrm{e} \AA^{-3}\right)$ & $0.37,-0.32$ \\
\hline
\end{tabular}

Computer programs: APEX3 (Bruker, 2016), SAINT-Plus (Bruker, 2016), SHELXS97 (Sheldrick, 2008), SHELXL2014/7 (Sheldrick, 2015), ORTEP-3 (Farrugia, 2012), Mercury 2020.1 (Macrae et al., 2020), SHELXTL (Sheldrick, 2008), PLATON (Spek, 2020), publCIF (Westrip, 2010).

$F_{\mathrm{o}}-F_{\mathrm{c}}$ map shows that the $\mathrm{H}$ atom could be residing part of the time on O41 and part of the time on N22.

\section{Acknowledgements}

CPG thanks SERB, New Delhi, for a project fellowship. This work was funded by the Science and Engineering Research Board (SERB), New Delhi (grant No. EEQ/2018/001172).

\section{Funding information}

Funding for this research was provided by: Science and Engineering Research Board (SERB), New Delhi (grant No. EEQ/2018/001172 to Dr. Rajesh G. Gonnade).

\section{References}

Braga, D., Dichiarante, E., Palladino, G., Grepioni, F., Chierotti, M. R., Gobetto, R. \& Pellegrino, L. (2010). CrystEngComm, 12, 3534-3536.

Braga, D., Giaffreda, S. L. \& Grepioni, F. (2006). Chem. Commun. pp. 3877-3879.

Bruker (2016). APEX3, SAINT-Plus and SADABS. Bruker AXS Inc., Madison, Wisconsin, USA.

Caspari, W. A. (1928). J. Chem. Soc. pp. 3235-3241.

Farrugia, L. J. (2012). J. Appl. Cryst. 45, 849-854.

Fitton, A. \& Goa, K. L. (1991). Drugs, 41, 780-798. 
Gilday, J. P., Graham, A. S., Ymen, B. I. \& Bohlin, M. (2005). US Patent US 2005/0209229 A1.

Gonnade, R. G. (2015). PCT Int. Appl. WO 2015/170345 A1.

Groom, C. R., Bruno, I. J., Lightfoot, M. P. \& Ward, S. C. (2016). Acta Cryst. B72, 171-179.

Grzesiak, M., Nitek, W., Rafalska-Łasocha, A. \& Łasocha, W. (2012). Z. Kristallogr. 227, 629-634.

Housty, J. \& Hospital, M. (1967). Acta Cryst. 22, 288-295.

Karki, S., Friščić, T. \& Jones, W. (2009). CrystEngComm, 11, 470481.

Kobayashi, K. \& Hagiwara, K. (2013). Targ Oncol, 8, 27-33.

Krueger, E. L., Sinha, A. S., Desper, J. \& Aakeröy, C. B. (2017). CrystEngComm, 19, 4605-4614.

Macrae, C. F., Sovago, I., Cottrell, S. J., Galek, P. T. A., McCabe, P., Pidcock, E., Platings, M., Shields, G. P., Stevens, J. S., Towler, M. \& Wood, P. A. (2020). J. Appl. Cryst. 53, 226-235.
Martins, I. C. B., Sardo, M., Santos, S. M., Fernandes, A., Antunes, A., André, V., Mafra, L. \& Duarte, M. T. (2016). Cryst. Growth Des. 16, 154-166.

Sheldrick, G. M. (2008). Acta Cryst. A64, 112-122.

Sheldrick, G. M. (2015). Acta Cryst. C71, 3-8.

Spek, A. L. (2020). Acta Cryst. E76, 1-11.

Thompson, L. J., Voguri, R. S., Male, L. \& Tremayne, M. (2011). CrystEngComm, 13, 4188-4195.

Thorat, S. H., Patwadkar, M. V., Gonnade, R. G. \& Vaidhyanathan, R. (2014). CrystEngComm, 16, 8638-8641.

Thorat, S. H., Sahu, S. K., Patwadkar, M. V., Badiger, M. V. \& Gonnade, R. G. (2015). J. Pharm. Sci. 104, 4207-4216.

Tothadi, S. \& Phadkule, A. (2019). CrystEngComm, 21, 2481-2484.

Westrip, S. P. (2010). J. Appl. Cryst. 43, 920-925.

Zhao, F.-H., Che, Y.-X. \& Zheng, J.-M. (2012). Cryst. Growth Des. 12 , 4712-4715. 


\section{supporting information}

Acta Cryst. (2020). E76, 884-888 [https://doi.org/10.1107/S2056989020006623]

\section{Crystal structure of a 1:1 co-crystal of the anticancer drug gefitinib with azelaic acid}

\section{Christy P. George, Ekta Sangtani and Rajesh G. Gonnade \\ Computing details}

Data collection: APEX3 (Bruker, 2016); cell refinement: SAINT-Plus (Bruker, 2016); data reduction: SAINT-Plus (Bruker, 2016); program(s) used to solve structure: SHELXS97 (Sheldrick, 2008); program(s) used to refine structure:

SHELXL2014/7 (Sheldrick, 2015); molecular graphics: ORTEP-3 (Farrugia, 2012), Mercury 2020.1 (Macrae et al., 2020); software used to prepare material for publication: SHELXTL (Sheldrick, 2008), PLATON (Spek, 2020), publCIF

(Westrip, 2010).

Quinazolin-4-amine-nonanedioic acid (1/1)

Crystal data

$\mathrm{C}_{22} \mathrm{H}_{24} \mathrm{ClFN}_{4} \mathrm{O}_{3} \cdot \mathrm{C}_{9} \mathrm{H}_{16} \mathrm{O}_{4}$

$M_{r}=635.12$

Monoclinic, $P 2_{1} / n$

$a=10.7716(10) \AA$

$b=7.4153(13) \AA$

$c=38.175(7) \AA$

$\beta=92.311(5)^{\circ}$

$V=3046.7(8) \AA^{3}$

$Z=4$

$$
F(000)=1344
$$

$D_{\mathrm{x}}=1.385 \mathrm{Mg} \mathrm{m}^{-3}$

Mo $K \alpha$ radiation, $\lambda=0.71073 \AA$

Cell parameters from 9964 reflections

$\theta=2.5-36.4^{\circ}$

$\mu=0.19 \mathrm{~mm}^{-1}$

$T=100 \mathrm{~K}$

Thin Needle, colourless

$0.28 \times 0.19 \times 0.04 \mathrm{~mm}$

Data collection

Bruker D8 VENTURE Kappa Duo PHOTON II CPAD

diffractometer

Radiation source: micro-focus sealed tube, Incoatech $\mathrm{I} \mu \mathrm{S} \mathrm{HB}$

Multilayer mirrors monochromator

Detector resolution: 7.39 pixels $\mathrm{mm}^{-1}$

$\varphi$ and $\omega$ scans

Absorption correction: multi-scan

(SADABS; Bruker, 2016)

Refinement

Refinement on $F^{2}$

Least-squares matrix: full

$R\left[F^{2}>2 \sigma\left(F^{2}\right)\right]=0.045$

$w R\left(F^{2}\right)=0.110$

$S=1.02$

7338 reflections

$T_{\min }=0.950, T_{\max }=0.993$

92337 measured reflections

7338 independent reflections

5413 reflections with $I>2 \sigma(I)$

$R_{\text {int }}=0.162$

$\theta_{\max }=28.0^{\circ}, \theta_{\min }=2.4^{\circ}$

$h=-14 \rightarrow 14$

$k=-9 \rightarrow 9$

$l=-50 \rightarrow 50$

\section{3 parameters}

126 restraints

Hydrogen site location: mixed

$\mathrm{H}$ atoms treated by a mixture of independent and constrained refinement 
$w=1 /\left[\sigma^{2}\left(F_{\mathrm{o}}^{2}\right)+(0.0458 P)^{2}+1.2854 P\right]$

where $P=\left(F_{\mathrm{o}}^{2}+2 F_{\mathrm{c}}^{2}\right) / 3$

$(\Delta / \sigma)_{\max }=0.001$

$$
\begin{aligned}
\Delta \rho_{\max } & =0.37 \mathrm{e} \AA^{-3} \\
\Delta \rho=-0.32 & \AA^{-3}
\end{aligned}
$$

\section{Special details}

Geometry. All esds (except the esd in the dihedral angle between two 1.s. planes) are estimated using the full covariance matrix. The cell esds are taken into account individually in the estimation of esds in distances, angles and torsion angles; correlations between esds in cell parameters are only used when they are defined by crystal symmetry. An approximate (isotropic) treatment of cell esds is used for estimating esds involving 1.s. planes.

Fractional atomic coordinates and isotropic or equivalent isotropic displacement parameters $\left(\AA^{2}\right)$

\begin{tabular}{|c|c|c|c|c|c|}
\hline & $x$ & $y$ & $z$ & $U_{\text {iso }} * / U_{\text {eq }}$ & Occ. $(<1)$ \\
\hline N1 & $0.47579(12)$ & $0.8204(2)$ & $0.55135(4)$ & 0.0133 & \\
\hline $\mathrm{C} 2$ & $0.36066(14)$ & $0.8699(2)$ & $0.54311(5)$ & $0.0148(4)$ & \\
\hline $\mathrm{H} 2$ & 0.3136 & 0.9129 & 0.5619 & $0.018 *$ & \\
\hline N3 & $0.30169(12)$ & $0.8670(2)$ & $0.51143(4)$ & $0.0144(3)$ & \\
\hline $\mathrm{C} 4$ & $0.36644(14)$ & $0.8072(2)$ & $0.48478(4)$ & 0.0113 & \\
\hline $\mathrm{C} 5$ & $0.56600(14)$ & $0.6768(2)$ & $0.46286(4)$ & $0.0111(3)$ & \\
\hline H5 & 0.5318 & 0.6671 & 0.4396 & $0.013 *$ & \\
\hline C6 & $0.68614(14)$ & $0.6245(2)$ & $0.47037(4)$ & $0.0109(3)$ & \\
\hline $\mathrm{C} 7$ & $0.73862(13)$ & $0.6397(2)$ & $0.50508(4)$ & $0.0116(3)$ & \\
\hline $\mathrm{C} 8$ & $0.66847(14)$ & $0.7041(2)$ & $0.53159(4)$ & $0.0117(3)$ & \\
\hline H8 & 0.7033 & 0.7129 & 0.5548 & $0.014 *$ & \\
\hline $\mathrm{C} 9$ & $0.54411(14)$ & $0.7573(2)$ & $0.52418(4)$ & $0.0115(3)$ & \\
\hline $\mathrm{C} 10$ & $0.49287(14)$ & $0.7456(2)$ & $0.48986(4)$ & 0.0108 & \\
\hline N11 & $0.31164(12)$ & $0.8064(2)$ & $0.45205(4)$ & 0.0129 & \\
\hline H11 & $0.3547(17)$ & $0.784(3)$ & $0.4348(5)$ & $0.018(5)^{*}$ & \\
\hline $\mathrm{C} 12$ & 0.1907 (2) & $0.8603(3)$ & $0.44138(7)$ & $0.0084(5)$ & $0.915(7)$ \\
\hline $\mathrm{C} 13$ & $0.1724(2)$ & $0.9202(3)$ & $0.40659(7)$ & $0.0103(5)$ & $0.915(7)$ \\
\hline H13 & 0.2409 & 0.9296 & 0.3918 & $0.012 *$ & $0.915(7)$ \\
\hline $\mathrm{C} 14$ & $0.0538(2)$ & 0.9655 & $0.39399(6)$ & $0.0126(5)$ & $0.915(7)$ \\
\hline C15 & $-0.0455(2)$ & $0.9516(5)$ & $0.41588(7)$ & $0.0121(6)$ & $0.915(7)$ \\
\hline C16 & $-0.0294(2)$ & 0.8968 (3) & $0.45021(8)$ & $0.0114(5)$ & $0.915(7)$ \\
\hline H16 & -0.0984 & 0.8906 & 0.4649 & $0.014 *$ & $0.915(7)$ \\
\hline $\mathrm{C} 17$ & $0.0893(2)$ & $0.8503(3)$ & $0.46331(6)$ & $0.0103(5)$ & $0.915(7)$ \\
\hline H17 & 0.1014 & 0.8120 & 0.4870 & $0.012 *$ & $0.915(7)$ \\
\hline $\mathrm{Cl1}$ & $0.03309(6)$ & $1.0446(3)$ & $0.35140(2)$ & 0.0239 & $0.915(7)$ \\
\hline F1 & $-0.1656(19)$ & $0.981(6)$ & $0.4079(9)$ & $0.0207(5)$ & $0.085(7)$ \\
\hline $\mathrm{C} 12^{\prime}$ & $0.165(3)$ & $0.869(4)$ & $0.4489(8)$ & $0.0084(5)$ & $0.085(7)$ \\
\hline C13' & $0.180(3)$ & $0.904(3)$ & $0.4171(9)$ & $0.0103(5)$ & $0.085(7)$ \\
\hline H13' & 0.2607 & 0.8997 & 0.4082 & $0.012 *$ & $0.085(7)$ \\
\hline $\mathrm{C} 14^{\prime}$ & $0.088(3)$ & $0.944(3)$ & $0.3964(9)$ & $0.0126(5)$ & $0.085(7)$ \\
\hline $\mathrm{C} 15^{\prime}$ & $-0.034(2)$ & $0.956(6)$ & $0.4083(11)$ & $0.0121(6)$ & $0.085(7)$ \\
\hline $\mathrm{C} 16^{\prime}$ & $-0.038(3)$ & $0.918(4)$ & $0.4387(10)$ & $0.0114(5)$ & $0.085(7)$ \\
\hline $\mathrm{H} 16^{\prime}$ & -0.1167 & 0.9307 & 0.4489 & $0.014 *$ & $0.085(7)$ \\
\hline $\mathrm{C} 17^{\prime}$ & $0.051(3)$ & $0.863(4)$ & $0.4588(8)$ & $0.0103(5)$ & $0.085(7)$ \\
\hline $\mathrm{H} 17^{\prime}$ & 0.0340 & 0.8158 & 0.4813 & $0.012 *$ & $0.085(7)$ \\
\hline $\mathrm{Cl}^{\prime}$ & $0.0350(8)$ & $0.977(3)$ & $0.3483(3)$ & 0.0239 & $0.085(7)$ \\
\hline
\end{tabular}




\begin{tabular}{|c|c|c|c|c|c|}
\hline $\mathrm{F} 1^{\prime}$ & $-0.15929(17)$ & $0.9946(5)$ & $0.40135(6)$ & $0.0207(5)$ & $0.915(7)$ \\
\hline $\mathrm{O} 18$ & $0.76536(10)$ & $0.55753(17)$ & $0.44664(3)$ & $0.0146(3)$ & \\
\hline $\mathrm{C} 19$ & $0.71844(14)$ & $0.5374(3)$ & $0.41114(4)$ & $0.0140(3)$ & \\
\hline H19A & 0.6840 & 0.6529 & 0.4021 & $0.017^{*}$ & \\
\hline H19B & 0.6523 & 0.4447 & 0.4096 & $0.017^{*}$ & \\
\hline $\mathrm{C} 20$ & $0.82895(15)$ & $0.4798(3)$ & $0.39031(5)$ & $0.0167(4)$ & \\
\hline $\mathrm{H} 20 \mathrm{~A}$ & 0.8851 & 0.5844 & 0.3879 & $0.020^{*}$ & \\
\hline $\mathrm{H} 20 \mathrm{~B}$ & 0.8755 & 0.3852 & 0.4036 & $0.020^{*}$ & \\
\hline $\mathrm{C} 21$ & $0.79365(15)$ & $0.4080(2)$ & $0.35412(5)$ & $0.0162(4)$ & \\
\hline $\mathrm{H} 21 \mathrm{~A}$ & 0.8708 & 0.3825 & 0.3418 & $0.019^{*}$ & \\
\hline $\mathrm{H} 21 \mathrm{~B}$ & 0.7492 & 0.2923 & 0.3567 & $0.019^{*}$ & \\
\hline N22 & $0.71453(13)$ & $0.5303(2)$ & 0.33193 (4) & $0.0170(3)$ & \\
\hline $\mathrm{C} 23$ & $0.69993(18)$ & $0.4460(3)$ & $0.29657(5)$ & $0.0252(4)$ & \\
\hline $\mathrm{H} 23 \mathrm{~A}$ & 0.6568 & 0.3287 & 0.2985 & $0.030^{*}$ & \\
\hline $\mathrm{H} 23 \mathrm{~B}$ & 0.7829 & 0.4231 & 0.2873 & $0.030^{*}$ & \\
\hline $\mathrm{C} 24$ & $0.6265(2)$ & $0.5668(4)$ & $0.27160(6)$ & $0.0392(6)$ & \\
\hline $\mathrm{H} 24 \mathrm{~A}$ & 0.6195 & 0.5091 & 0.2482 & $0.047^{*}$ & \\
\hline H24B & 0.5415 & 0.5827 & 0.2801 & $0.047^{*}$ & \\
\hline $\mathrm{O} 25$ & $0.68388(16)$ & $0.7383(3)$ & $0.26857(4)$ & $0.0467(5)$ & \\
\hline $\mathrm{C} 26$ & $0.6932(2)$ & $0.8207(3)$ & $0.30238(6)$ & $0.0426(6)$ & \\
\hline $\mathrm{H} 26 \mathrm{~A}$ & 0.6088 & 0.8361 & 0.3113 & $0.051^{*}$ & \\
\hline $\mathrm{H} 26 \mathrm{~B}$ & 0.7306 & 0.9419 & 0.3002 & $0.051^{*}$ & \\
\hline $\mathrm{C} 27$ & $0.77011(19)$ & $0.7117(3)$ & $0.32823(5)$ & $0.0261(4)$ & \\
\hline $\mathrm{H} 27 \mathrm{~A}$ & 0.8558 & 0.7002 & 0.3201 & $0.031^{*}$ & \\
\hline $\mathrm{H} 27 \mathrm{~B}$ & 0.7740 & 0.7734 & 0.3512 & $0.031^{*}$ & \\
\hline $\mathrm{O} 28$ & $0.85921(10)$ & $0.58683(17)$ & $0.50862(3)$ & $0.0148(3)$ & \\
\hline $\mathrm{C} 29$ & $0.92077(14)$ & $0.6098(3)$ & $0.54230(5)$ & $0.0162(4)$ & \\
\hline H29A & 0.9179 & 0.7371 & 0.5491 & $0.024 *$ & \\
\hline H29B & 1.0075 & 0.5712 & 0.5412 & $0.024 *$ & \\
\hline $\mathrm{H} 29 \mathrm{C}$ & 0.8790 & 0.5367 & 0.5597 & $0.024 *$ & \\
\hline $\mathrm{O} 30$ & $0.58731(10)$ & $0.86845(18)$ & $0.61271(3)$ & $0.0181(3)$ & \\
\hline $\mathrm{H} 30$ & $0.533(2)$ & $0.852(4)$ & $0.5915(7)$ & $0.056(8)^{*}$ & \\
\hline O31 & $0.41210(11)$ & $0.90780(19)$ & $0.64135(4)$ & $0.0238(3)$ & \\
\hline C32 & $0.52448(15)$ & $0.9063(2)$ & $0.64073(5)$ & $0.0169(4)$ & \\
\hline $\mathrm{C} 33$ & $0.60896(16)$ & $0.9548(3)$ & $0.67194(5)$ & $0.0209(4)$ & \\
\hline H33A & 0.6142 & 1.0879 & 0.6735 & $0.025^{*}$ & \\
\hline H33B & 0.6934 & 0.9093 & 0.6676 & $0.025^{*}$ & \\
\hline C34 & $0.57017(16)$ & $0.8827(3)$ & $0.70716(5)$ & $0.0200(4)$ & \\
\hline $\mathrm{H} 34 \mathrm{~A}$ & 0.5747 & 0.7494 & 0.7073 & $0.024 *$ & \\
\hline H34B & 0.4834 & 0.9184 & 0.7112 & $0.024 *$ & \\
\hline C35 & $0.6584(3)$ & $0.9608(5)$ & $0.73679(8)$ & $0.0196(6)$ & $0.770(4)$ \\
\hline $\mathrm{H} 35 \mathrm{~A}$ & 0.7449 & 0.9243 & 0.7324 & $0.023^{*}$ & $0.770(4)$ \\
\hline H35B & 0.6546 & 1.0941 & 0.7360 & $0.023^{*}$ & $0.770(4)$ \\
\hline C36 & $0.6250(3)$ & 0.8968 (3) & $0.77317(6)$ & $0.0178(6)$ & $0.770(4)$ \\
\hline $\mathrm{H} 36 \mathrm{~A}$ & 0.6289 & 0.7634 & 0.7740 & $0.021^{*}$ & $0.770(4)$ \\
\hline H36B & 0.5388 & 0.9335 & 0.7777 & $0.021^{*}$ & $0.770(4)$ \\
\hline C37 & $0.7126(2)$ & $0.9744(4)$ & $0.80178(8)$ & $0.0186(6)$ & $0.770(4)$ \\
\hline H37A & 0.7989 & 0.9407 & 0.7967 & $0.022 *$ & $0.770(4)$ \\
\hline
\end{tabular}




\begin{tabular}{|c|c|c|c|c|c|}
\hline H37B & 0.7070 & 1.1077 & 0.8011 & $0.022 *$ & $0.770(4)$ \\
\hline C38 & $0.6847(2)$ & $0.9101(3)$ & $0.83854(7)$ & $0.0174(6)$ & $0.770(4)$ \\
\hline H38A & 0.6933 & 0.7772 & 0.8394 & $0.021 *$ & $0.770(4)$ \\
\hline H38B & 0.5973 & 0.9397 & 0.8432 & $0.021 *$ & $0.770(4)$ \\
\hline C39 & $0.7695(3)$ & $0.9931(5)$ & $0.86770(8)$ & $0.0150(7)$ & $0.770(4)$ \\
\hline H39A & 0.7688 & 1.1260 & 0.8653 & $0.018^{*}$ & $0.770(4)$ \\
\hline H39B & 0.7366 & 0.9625 & 0.8908 & $0.018^{*}$ & $0.770(4)$ \\
\hline $\mathrm{C} 35^{\prime}$ & $0.6128(9)$ & 0.9559 (19) & 0.7400 & $0.0196(6)$ & $0.230(4)$ \\
\hline $\mathrm{H} 35 \mathrm{C}$ & 0.6048 & 1.0888 & 0.7387 & $0.023 *$ & $0.230(4)$ \\
\hline H35D & 0.7025 & 0.9281 & 0.7433 & $0.023^{*}$ & $0.230(4)$ \\
\hline C36' & $0.5482(8)$ & $0.8921(13)$ & $0.7725(2)$ & $0.023(2)$ & $0.230(4)$ \\
\hline $\mathrm{H} 36 \mathrm{C}$ & 0.5562 & 0.7594 & 0.7743 & $0.027 *$ & $0.230(4)$ \\
\hline H36D & 0.4586 & 0.9209 & 0.7698 & $0.027^{*}$ & $0.230(4)$ \\
\hline C37' & $0.6007(7)$ & $0.9780(13)$ & $0.8072(2)$ & $0.023(2)$ & $0.230(4)$ \\
\hline $\mathrm{H} 37 \mathrm{C}$ & 0.5988 & 1.1109 & 0.8048 & $0.028^{*}$ & $0.230(4)$ \\
\hline H37D & 0.5456 & 0.9453 & 0.8264 & $0.028 *$ & $0.230(4)$ \\
\hline C $38^{\prime}$ & $0.7281(8)$ & $0.9218(12)$ & 0.8171 & 0.0139 (19) & $0.230(4)$ \\
\hline $\mathrm{H} 38 \mathrm{C}$ & 0.7850 & 0.9748 & 0.8002 & $0.017 *$ & $0.230(4)$ \\
\hline H38D & 0.7333 & 0.7890 & 0.8149 & $0.017 *$ & $0.230(4)$ \\
\hline C39' & $0.7737(12)$ & 0.9740 (19) & 0.8538 & $0.019(2)$ & $0.230(4)$ \\
\hline H39C & 0.7657 & 1.1065 & 0.8559 & $0.023 *$ & $0.230(4)$ \\
\hline H39D & 0.7161 & 0.9199 & 0.8705 & $0.023 *$ & $0.230(4)$ \\
\hline $\mathrm{C} 40$ & $0.90344(15)$ & $0.9248(2)$ & $0.86617(5)$ & $0.0153(4)$ & \\
\hline O41 & $0.97487(11)$ & $1.00506(19)$ & $0.84437(3)$ & $0.0235(3)$ & \\
\hline H41 & $1.060(2)$ & $0.950(2)$ & $0.8463(4)$ & $0.035^{*}$ & \\
\hline $\mathrm{O} 42$ & $0.93796(11)$ & 0.80413 (19) & $0.88602(3)$ & 0.0224 & \\
\hline
\end{tabular}

Atomic displacement parameters $\left(\AA^{2}\right)$

\begin{tabular}{lllllll}
\hline & $U^{11}$ & $U^{22}$ & $U^{33}$ & $U^{12}$ & $U^{13}$ & $U^{23}$ \\
\hline N1 & $0.0133(6)$ & $0.0141(7)$ & $0.0125(7)$ & $0.0010(6)$ & $0.0024(5)$ & $-0.0003(6)$ \\
C2 & $0.0139(7)$ & $0.0173(9)$ & $0.0133(8)$ & $0.0029(7)$ & $0.0036(6)$ & $-0.0007(7)$ \\
N3 & $0.0127(6)$ & $0.0165(8)$ & $0.0140(7)$ & $0.0020(6)$ & $0.0015(5)$ & $0.0003(6)$ \\
C4 & $0.0119(7)$ & $0.0092(8)$ & $0.0128(8)$ & $-0.0007(6)$ & $0.0005(6)$ & $0.0004(7)$ \\
C5 & $0.0106(7)$ & $0.0123(8)$ & $0.0101(8)$ & $-0.0005(6)$ & $-0.0005(6)$ & $0.0001(7)$ \\
C6 & $0.0109(7)$ & $0.0106(8)$ & $0.0114(8)$ & $-0.0002(6)$ & $0.0027(6)$ & $0.0000(7)$ \\
C7 & $0.0088(7)$ & $0.0100(8)$ & $0.0160(9)$ & $-0.0006(6)$ & $-0.0011(6)$ & $0.0015(7)$ \\
C8 & $0.0126(7)$ & $0.0116(8)$ & $0.0107(8)$ & $-0.0007(6)$ & $-0.0023(6)$ & $0.0007(7)$ \\
C9 & $0.0127(7)$ & $0.0090(8)$ & $0.0129(8)$ & $-0.0001(6)$ & $0.0020(6)$ & $-0.0001(7)$ \\
C10 & $0.0112(7)$ & $0.0078(8)$ & $0.0135(8)$ & $0.0005(6)$ & $0.0012(6)$ & $0.0011(6)$ \\
N11 & $0.0076(6)$ & $0.0195(8)$ & $0.0118(7)$ & $0.0034(6)$ & $0.0017(5)$ & $-0.0015(6)$ \\
C12 & $0.0041(10)$ & $0.0098(8)$ & $0.0114(13)$ & $0.0021(7)$ & $0.0028(7)$ & $-0.0018(8)$ \\
C13 & $0.0085(8)$ & $0.0165(10)$ & $0.0059(12)$ & $0.0021(7)$ & $0.0002(9)$ & $0.0017(8)$ \\
C14 & $0.0071(11)$ & $0.0177(10)$ & $0.0127(9)$ & $0.0050(8)$ & $-0.0034(9)$ & $0.0015(8)$ \\
C15 & $0.0086(8)$ & $0.0157(9)$ & $0.0120(17)$ & $0.0024(7)$ & $-0.0011(7)$ & $-0.0018(11)$ \\
C16 & $0.0083(8)$ & $0.0165(10)$ & $0.0093(12)$ & $0.0008(7)$ & $0.0016(9)$ & $-0.0005(9)$ \\
C17 & $0.0035(10)$ & $0.0140(9)$ & $0.0132(9)$ & $0.0011(8)$ & $-0.0014(8)$ & $0.0002(7)$ \\
C11 & $0.01550(19)$ & $0.0375(8)$ & $0.0185(3)$ & $0.0082(3)$ & $-0.00091(18)$ & $0.0091(4)$
\end{tabular}




\begin{tabular}{|c|c|c|c|c|c|c|}
\hline $\mathrm{F} 1$ & $0.0077(5)$ & $0.0352(9)$ & $0.0190(13)$ & $0.0063(5)$ & $-0.0013(6)$ & $-0.0007(9)$ \\
\hline $\mathrm{C} 12^{\prime}$ & $0.0041(10)$ & $0.0098(8)$ & $0.0114(13)$ & $0.0021(7)$ & $0.0028(7)$ & $-0.0018(8)$ \\
\hline $\mathrm{C} 13^{\prime}$ & $0.0085(8)$ & $0.0165(10)$ & $0.0059(12)$ & $0.0021(7)$ & $0.0002(9)$ & $0.0017(8)$ \\
\hline $\mathrm{C} 14^{\prime}$ & $0.0071(11)$ & $0.0177(10)$ & $0.0127(9)$ & $0.0050(8)$ & $-0.0034(9)$ & $0.0015(8)$ \\
\hline $\mathrm{C} 15^{\prime}$ & $0.0086(8)$ & $0.0157(9)$ & $0.0120(17)$ & $0.0024(7)$ & $-0.0011(7)$ & $-0.0018(11)$ \\
\hline $\mathrm{C} 16^{\prime}$ & $0.0083(8)$ & $0.0165(10)$ & $0.0093(12)$ & $0.0008(7)$ & $0.0016(9)$ & $-0.0005(9)$ \\
\hline $\mathrm{C} 17^{\prime}$ & $0.0035(10)$ & $0.0140(9)$ & $0.0132(9)$ & $0.0011(8)$ & $-0.0014(8)$ & $0.0002(7)$ \\
\hline $\mathrm{Cl1}^{\prime}$ & 0.01550 (19) & $0.0375(8)$ & $0.0185(3)$ & $0.0082(3)$ & $-0.00091(18)$ & $0.0091(4)$ \\
\hline $\mathrm{F} 1^{\prime}$ & $0.0077(5)$ & $0.0352(9)$ & $0.0190(13)$ & $0.0063(5)$ & $-0.0013(6)$ & $-0.0007(9)$ \\
\hline $\mathrm{O} 18$ & $0.0105(5)$ & $0.0228(7)$ & $0.0106(6)$ & $0.0042(5)$ & $-0.0001(4)$ & $-0.0030(5)$ \\
\hline C19 & $0.0114(7)$ & $0.0206(9)$ & $0.0098(8)$ & $0.0009(7)$ & $-0.0015(6)$ & $-0.0007(7)$ \\
\hline $\mathrm{C} 20$ & $0.0121(7)$ & $0.0224(10)$ & $0.0156(9)$ & $0.0028(7)$ & $0.0009(6)$ & $-0.0040(8)$ \\
\hline $\mathrm{C} 21$ & $0.0169(8)$ & $0.0157(9)$ & $0.0160(9)$ & $0.0040(7)$ & $0.0016(7)$ & $-0.0010(7)$ \\
\hline $\mathrm{N} 22$ & $0.0211(7)$ & $0.0188(8)$ & $0.0113(7)$ & $0.0053(6)$ & $0.0028(6)$ & $-0.0004(6)$ \\
\hline $\mathrm{C} 23$ & $0.0275(9)$ & $0.0347(12)$ & $0.0133(9)$ & $0.0098(9)$ & $0.0015(7)$ & $-0.0053(9)$ \\
\hline $\mathrm{C} 24$ & $0.0400(12)$ & $0.0615(17)$ & $0.0161(10)$ & $0.0193(12)$ & $0.0034(9)$ & $0.0049(11)$ \\
\hline $\mathrm{O} 25$ & $0.0590(10)$ & $0.0591(12)$ & $0.0235(9)$ & $0.0253(9)$ & $0.0179(7)$ & $0.0200(8)$ \\
\hline $\mathrm{C} 26$ & $0.0637(15)$ & $0.0314(13)$ & $0.0347(14)$ & $0.0212(12)$ & $0.0252(12)$ & $0.0158(11)$ \\
\hline $\mathrm{C} 27$ & $0.0396(11)$ & $0.0170(10)$ & $0.0228(11)$ & $0.0020(8)$ & $0.0143(9)$ & $0.0010(8)$ \\
\hline $\mathrm{O} 28$ & $0.0096(5)$ & $0.0212(7)$ & $0.0135(6)$ & $0.0038(5)$ & -0.0025 & $-0.0022(5)$ \\
\hline $\mathrm{C} 29$ & $0.0123(7)$ & $0.0214(10)$ & $0.0145(9)$ & $0.0028(7)$ & $-0.0052(6)$ & $-0.0036(7)$ \\
\hline $\mathrm{O} 30$ & $0.0150(5)$ & $0.0276(7)$ & $0.0116(6)$ & $0.0001(5)$ & -0.0004 & $-0.0023(6)$ \\
\hline $\mathrm{O} 31$ & $0.0141(6)$ & $0.0344(8)$ & $0.0232(7)$ & $-0.0014(5)$ & $0.0020(5)$ & $-0.0030(6)$ \\
\hline $\mathrm{C} 32$ & $0.0174(8)$ & $0.0181(9)$ & $0.0153(9)$ & $-0.0011(7)$ & $0.0011(7)$ & $0.0000(7)$ \\
\hline $\mathrm{C} 33$ & $0.0192(8)$ & $0.0276(11)$ & $0.0159(9)$ & $-0.0043(8)$ & $-0.0005(7)$ & $-0.0023(8)$ \\
\hline $\mathrm{C} 34$ & $0.0254(9)$ & $0.0197(10)$ & $0.0148(9)$ & $0.0011(8)$ & $-0.0001(7)$ & $-0.0006(8)$ \\
\hline $\mathrm{C} 35$ & $0.0201(15)$ & $0.0227(11)$ & $0.0159(12)$ & $-0.0061(15)$ & $0.0000(13)$ & $-0.0001(9)$ \\
\hline $\mathrm{C} 36$ & $0.0162(13)$ & $0.0209(13)$ & $0.0161(12)$ & $-0.0027(10)$ & $-0.0023(10)$ & $-0.0003(10)$ \\
\hline $\mathrm{C} 37$ & $0.0196(12)$ & $0.0229(15)$ & $0.0133(14)$ & $-0.0058(11)$ & $-0.0012(11)$ & $0.0028(12)$ \\
\hline C38 & $0.0125(10)$ & $0.0247(14)$ & $0.0149(14)$ & $-0.0037(9)$ & $-0.0005(10)$ & $0.0013(10)$ \\
\hline C39 & $0.0134(11)$ & $0.0207(15)$ & $0.0111(16)$ & $-0.0003(10)$ & $0.0009(14)$ & $-0.0017(15)$ \\
\hline $\mathrm{C} 35^{\prime}$ & $0.0201(15)$ & $0.0227(11)$ & $0.0159(12)$ & $-0.0061(15)$ & $0.0000(13)$ & $-0.0001(9)$ \\
\hline $\mathrm{C} 36^{\prime}$ & $0.014(5)$ & $0.035(5)$ & $0.019(4)$ & $0.002(4)$ & $0.001(3)$ & $-0.003(4)$ \\
\hline $\mathrm{C} 37^{\prime}$ & $0.015(4)$ & $0.033(5)$ & $0.021(5)$ & $0.000(3)$ & $-0.002(3)$ & $-0.006(4)$ \\
\hline $\mathrm{C} 38^{\prime}$ & $0.017(4)$ & $0.013(4)$ & $0.011(5)$ & -0.002 & $-0.004(4)$ & $0.001(4)$ \\
\hline $\mathrm{C} 39^{\prime}$ & $0.023(4)$ & $0.021(5)$ & $0.014(6)$ & $0.003(3)$ & $0.010(5)$ & $-0.005(5)$ \\
\hline $\mathrm{C} 40$ & $0.0153(7)$ & $0.0154(9)$ & $0.0148(9)$ & $-0.0042(7)$ & $-0.0028(7)$ & $-0.0027(7)$ \\
\hline $\mathrm{O} 41$ & $0.0185(6)$ & $0.0302(8)$ & $0.0215(7)$ & $-0.0046(6)$ & $-0.0012(5)$ & $0.0094(6)$ \\
\hline $\mathrm{O} 42$ & $0.0171(6)$ & $0.0288(8)$ & $0.0209(7)$ & $-0.0047(5)$ & $-0.0044(5)$ & $0.0085(6)$ \\
\hline
\end{tabular}

Geometric parameters $(\AA, \stackrel{\circ}{)})$

\begin{tabular}{llll}
\hline $\mathrm{N} 1-\mathrm{C} 2$ & $1.319(2)$ & $\mathrm{C} 24-\mathrm{O} 25$ & $1.421(3)$ \\
$\mathrm{N} 1-\mathrm{C} 9$ & $1.378(2)$ & $\mathrm{C} 24-\mathrm{H} 24 \mathrm{~A}$ & 0.9900 \\
$\mathrm{C} 2-\mathrm{N} 3$ & $1.343(2)$ & $\mathrm{C} 24-\mathrm{H} 24 \mathrm{~B}$ & 0.9900 \\
$\mathrm{C} 2-\mathrm{H} 2$ & 0.9500 & $\mathrm{O} 25-\mathrm{C} 26$ & $1.428(3)$ \\
$\mathrm{N} 3-\mathrm{C} 4$ & $1.333(2)$ & $\mathrm{C} 26-\mathrm{C} 27$ & $0.499(3)$ \\
$\mathrm{C} 4-\mathrm{N} 11$ & $1.360(2)$ & $\mathrm{C} 26-\mathrm{H} 26 \mathrm{~A}$ & 0.9900
\end{tabular}




\begin{tabular}{|c|c|c|c|}
\hline $\mathrm{C} 4-\mathrm{C} 10$ & $1.442(2)$ & $\mathrm{C} 26-\mathrm{H} 26 \mathrm{~B}$ & 0.9900 \\
\hline $\mathrm{C} 5-\mathrm{C} 6$ & $1.370(2)$ & $\mathrm{C} 27-\mathrm{H} 27 \mathrm{~A}$ & 0.9900 \\
\hline $\mathrm{C} 5-\mathrm{C} 10$ & 1.417 (2) & $\mathrm{C} 27-\mathrm{H} 27 \mathrm{~B}$ & 0.9900 \\
\hline $\mathrm{C} 5-\mathrm{H} 5$ & 0.9500 & $\mathrm{O} 28-\mathrm{C} 29$ & $1.433(2)$ \\
\hline $\mathrm{C} 6-\mathrm{O} 18$ & $1.3631(19)$ & $\mathrm{C} 29-\mathrm{H} 29 \mathrm{~A}$ & 0.9800 \\
\hline $\mathrm{C} 6-\mathrm{C} 7$ & $1.424(2)$ & $\mathrm{C} 29-\mathrm{H} 29 \mathrm{~B}$ & 0.9800 \\
\hline $\mathrm{C} 7-\mathrm{O} 28$ & $1.3585(18)$ & $\mathrm{C} 29-\mathrm{H} 29 \mathrm{C}$ & 0.9800 \\
\hline $\mathrm{C} 7-\mathrm{C} 8$ & $1.373(2)$ & $\mathrm{O} 30-\mathrm{C} 32$ & $1.319(2)$ \\
\hline $\mathrm{C} 8-\mathrm{C} 9$ & $1.414(2)$ & $\mathrm{O} 30-\mathrm{H} 30$ & $0.99(3)$ \\
\hline $\mathrm{C} 8-\mathrm{H} 8$ & 0.9500 & $\mathrm{O} 31-\mathrm{C} 32$ & $1.212(2)$ \\
\hline $\mathrm{C} 9-\mathrm{C} 10$ & $1.404(2)$ & $\mathrm{C} 32-\mathrm{C} 33$ & $1.513(2)$ \\
\hline $\mathrm{N} 11-\mathrm{C} 12$ & $1.408(2)$ & $\mathrm{C} 33-\mathrm{C} 34$ & $1.521(3)$ \\
\hline $\mathrm{N} 11-\mathrm{C} 12^{\prime}$ & $1.64(3)$ & C33-H33A & 0.9900 \\
\hline N11-H11 & $0.84(2)$ & С $33-\mathrm{H} 33 \mathrm{~B}$ & 0.9900 \\
\hline $\mathrm{C} 12-\mathrm{C} 17$ & $1.404(3)$ & $\mathrm{C} 34-\mathrm{C} 35^{\prime}$ & $1.426(12)$ \\
\hline $\mathrm{C} 12-\mathrm{C} 13$ & $1.407(3)$ & C34-C35 & $1.559(4)$ \\
\hline $\mathrm{C} 13-\mathrm{C} 14$ & $1.389(3)$ & $\mathrm{C} 34-\mathrm{H} 34 \mathrm{~A}$ & 0.9900 \\
\hline $\mathrm{C} 13-\mathrm{H} 13$ & 0.9500 & С $34-\mathrm{H} 34 \mathrm{~B}$ & 0.9900 \\
\hline $\mathrm{C} 14-\mathrm{C} 15$ & $1.387(3)$ & $\mathrm{C} 35-\mathrm{C} 36$ & $1.525(4)$ \\
\hline $\mathrm{C} 14-\mathrm{Cl1}$ & $1.734(3)$ & $\mathrm{C} 35-\mathrm{H} 35 \mathrm{~A}$ & 0.9900 \\
\hline $\mathrm{C} 15-\mathrm{F} 1$ & 1.335 (19) & С $35-\mathrm{H} 35 \mathrm{~B}$ & 0.9900 \\
\hline $\mathrm{C} 15-\mathrm{C} 16$ & $1.377(3)$ & $\mathrm{C} 36-\mathrm{C} 37$ & $1.527(4)$ \\
\hline $\mathrm{C} 16-\mathrm{C} 17$ & $1.397(3)$ & С $36-\mathrm{H} 36 \mathrm{~A}$ & 0.9900 \\
\hline C16-H16 & 0.9500 & $\mathrm{C} 36-\mathrm{H} 36 \mathrm{~B}$ & 0.9900 \\
\hline $\mathrm{C} 17-\mathrm{H} 17$ & 0.9500 & $\mathrm{C} 37-\mathrm{C} 38$ & $1.524(4)$ \\
\hline $\mathrm{C} 12^{\prime}-\mathrm{C} 13^{\prime}$ & $1.26(4)$ & С $37-\mathrm{H} 37 \mathrm{~A}$ & 0.9900 \\
\hline $\mathrm{C} 12^{\prime}-\mathrm{C} 17^{\prime}$ & $1.31(3)$ & С $37-\mathrm{H} 37 \mathrm{~B}$ & 0.9900 \\
\hline $\mathrm{C} 13^{\prime}-\mathrm{C} 14^{\prime}$ & $1.27(4)$ & $\mathrm{C} 38-\mathrm{C} 39$ & $1.540(4)$ \\
\hline $\mathrm{C} 13^{\prime}-\mathrm{H} 13^{\prime}$ & 0.9500 & C $38-\mathrm{H} 38 \mathrm{~A}$ & 0.9900 \\
\hline $\mathrm{C} 14^{\prime}-\mathrm{C} 15^{\prime}$ & $1.41(4)$ & С $38-\mathrm{H} 38 \mathrm{~B}$ & 0.9900 \\
\hline $\mathrm{C} 14^{\prime}-\mathrm{Cl1}^{\prime}$ & $1.92(3)$ & $\mathrm{C} 39-\mathrm{C} 40$ & $1.533(4)$ \\
\hline $\mathrm{C} 15^{\prime}-\mathrm{C} 16^{\prime}$ & $1.20(4)$ & С $39-\mathrm{H} 39 \mathrm{~A}$ & 0.9900 \\
\hline $\mathrm{C} 15^{\prime}-\mathrm{F} 1^{\prime}$ & 1.395 (19) & С $39-$ Н39В & 0.9900 \\
\hline $\mathrm{C} 16^{\prime}-\mathrm{C} 17^{\prime}$ & $1.27(4)$ & $\mathrm{C} 35^{\prime}-\mathrm{C} 36^{\prime}$ & $1.522(14)$ \\
\hline $\mathrm{C} 16^{\prime}-\mathrm{H} 16^{\prime}$ & 0.9500 & $\mathrm{C} 35^{\prime}-\mathrm{H} 35 \mathrm{C}$ & 0.9900 \\
\hline $\mathrm{C} 17^{\prime}-\mathrm{H} 17^{\prime}$ & 0.9500 & $\mathrm{C} 35^{\prime}-\mathrm{H} 35 \mathrm{D}$ & 0.9900 \\
\hline $\mathrm{O} 18-\mathrm{C} 19$ & $1.435(2)$ & $\mathrm{C} 36^{\prime}-\mathrm{C} 37^{\prime}$ & $1.556(12)$ \\
\hline $\mathrm{C} 19-\mathrm{C} 20$ & $1.519(2)$ & $\mathrm{C} 36^{\prime}-\mathrm{H} 36 \mathrm{C}$ & 0.9900 \\
\hline C19-H19A & 0.9900 & $\mathrm{C} 36^{\prime}-\mathrm{H} 36 \mathrm{D}$ & 0.9900 \\
\hline C19-H19B & 0.9900 & $\mathrm{C} 37^{\prime}-\mathrm{C} 38^{\prime}$ & $1.469(11)$ \\
\hline $\mathrm{C} 20-\mathrm{C} 21$ & $1.515(2)$ & $\mathrm{C} 37^{\prime}-\mathrm{H} 37 \mathrm{C}$ & 0.9900 \\
\hline $\mathrm{C} 20-\mathrm{H} 20 \mathrm{~A}$ & 0.9900 & $\mathrm{C} 37^{\prime}-\mathrm{H} 37 \mathrm{D}$ & 0.9900 \\
\hline $\mathrm{C} 20-\mathrm{H} 20 \mathrm{~B}$ & 0.9900 & $\mathrm{C} 38^{\prime}-\mathrm{C} 39^{\prime}$ & $1.516(15)$ \\
\hline $\mathrm{C} 21-\mathrm{N} 22$ & $1.486(2)$ & $\mathrm{C} 38^{\prime}-\mathrm{H} 38 \mathrm{C}$ & 0.9900 \\
\hline $\mathrm{C} 21-\mathrm{H} 21 \mathrm{~A}$ & 0.9900 & $\mathrm{C} 38^{\prime}-\mathrm{H} 38 \mathrm{D}$ & 0.9900 \\
\hline $\mathrm{C} 21-\mathrm{H} 21 \mathrm{~B}$ & 0.9900 & $\mathrm{C} 39^{\prime}-\mathrm{C} 40$ & $1.501(13)$ \\
\hline $\mathrm{N} 22-\mathrm{C} 27$ & $1.482(2)$ & $\mathrm{C} 39^{\prime}-\mathrm{H} 39 \mathrm{C}$ & 0.9900 \\
\hline $\mathrm{N} 22-\mathrm{C} 23$ & $1.490(2)$ & C39'-H39D & 0.9900 \\
\hline
\end{tabular}




\begin{tabular}{|c|c|}
\hline $\mathrm{C} 23-\mathrm{C} 24$ & $1.509(3)$ \\
\hline $\mathrm{C} 23-\mathrm{H} 23 \mathrm{~A}$ & 0.9900 \\
\hline $\mathrm{C} 23-\mathrm{H} 23 \mathrm{~B}$ & 0.9900 \\
\hline $\mathrm{C} 2-\mathrm{N} 1-\mathrm{C} 9$ & $116.13(14)$ \\
\hline $\mathrm{N} 1-\mathrm{C} 2-\mathrm{N} 3$ & $128.05(15)$ \\
\hline $\mathrm{N} 1-\mathrm{C} 2-\mathrm{H} 2$ & 116.0 \\
\hline $\mathrm{N} 3-\mathrm{C} 2-\mathrm{H} 2$ & 116.0 \\
\hline $\mathrm{C} 4-\mathrm{N} 3-\mathrm{C} 2$ & $116.72(14)$ \\
\hline $\mathrm{N} 3-\mathrm{C} 4-\mathrm{N} 11$ & $118.80(14)$ \\
\hline $\mathrm{N} 3-\mathrm{C} 4-\mathrm{C} 10$ & $121.53(15)$ \\
\hline $\mathrm{N} 11-\mathrm{C} 4-\mathrm{C} 10$ & $119.66(14)$ \\
\hline $\mathrm{C} 6-\mathrm{C} 5-\mathrm{C} 10$ & $119.83(15)$ \\
\hline $\mathrm{C} 6-\mathrm{C} 5-\mathrm{H} 5$ & 120.1 \\
\hline $\mathrm{C} 10-\mathrm{C} 5-\mathrm{H} 5$ & 120.1 \\
\hline $\mathrm{O} 18-\mathrm{C} 6-\mathrm{C} 5$ & $125.13(15)$ \\
\hline $\mathrm{O} 18-\mathrm{C} 6-\mathrm{C} 7$ & $114.31(13)$ \\
\hline $\mathrm{C} 5-\mathrm{C} 6-\mathrm{C} 7$ & $120.56(14)$ \\
\hline $\mathrm{O} 28-\mathrm{C} 7-\mathrm{C} 8$ & $125.43(15)$ \\
\hline $\mathrm{O} 28-\mathrm{C} 7-\mathrm{C} 6$ & $114.31(14)$ \\
\hline $\mathrm{C} 8-\mathrm{C} 7-\mathrm{C} 6$ & $120.26(14)$ \\
\hline $\mathrm{C} 7-\mathrm{C} 8-\mathrm{C} 9$ & $119.64(15)$ \\
\hline $\mathrm{C} 7-\mathrm{C} 8-\mathrm{H} 8$ & 120.2 \\
\hline $\mathrm{C} 9-\mathrm{C} 8-\mathrm{H} 8$ & 120.2 \\
\hline $\mathrm{N} 1-\mathrm{C} 9-\mathrm{C} 10$ & $121.46(14)$ \\
\hline $\mathrm{N} 1-\mathrm{C} 9-\mathrm{C} 8$ & $118.33(15)$ \\
\hline $\mathrm{C} 10-\mathrm{C} 9-\mathrm{C} 8$ & $120.21(14)$ \\
\hline $\mathrm{C} 9-\mathrm{C} 10-\mathrm{C} 5$ & $119.48(14)$ \\
\hline $\mathrm{C} 9-\mathrm{C} 10-\mathrm{C} 4$ & $116.09(14)$ \\
\hline $\mathrm{C} 5-\mathrm{C} 10-\mathrm{C} 4$ & $124.43(15)$ \\
\hline $\mathrm{C} 4-\mathrm{N} 11-\mathrm{C} 12$ & $128.84(17)$ \\
\hline $\mathrm{C} 4-\mathrm{N} 11-\mathrm{C} 12^{\prime}$ & $116.5(11)$ \\
\hline $\mathrm{C} 4-\mathrm{N} 11-\mathrm{H} 11$ & $119.4(13)$ \\
\hline $\mathrm{C} 12-\mathrm{N} 11-\mathrm{H} 11$ & $111.4(13)$ \\
\hline $\mathrm{C} 12^{\prime}-\mathrm{N} 11-\mathrm{H} 11$ & $123.9(17)$ \\
\hline $\mathrm{C} 17-\mathrm{C} 12-\mathrm{C} 13$ & $119.82(19)$ \\
\hline $\mathrm{C} 17-\mathrm{C} 12-\mathrm{N} 11$ & $123.1(3)$ \\
\hline $\mathrm{C} 13-\mathrm{C} 12-\mathrm{N} 11$ & $117.1(2)$ \\
\hline $\mathrm{C} 14-\mathrm{C} 13-\mathrm{C} 12$ & 119.7 (2) \\
\hline $\mathrm{C} 14-\mathrm{C} 13-\mathrm{H} 13$ & 120.2 \\
\hline $\mathrm{C} 12-\mathrm{C} 13-\mathrm{H} 13$ & 120.2 \\
\hline $\mathrm{C} 15-\mathrm{C} 14-\mathrm{C} 13$ & $119.6(2)$ \\
\hline $\mathrm{C} 15-\mathrm{C} 14-\mathrm{Cl1}$ & $121.1(2)$ \\
\hline $\mathrm{C} 13-\mathrm{C} 14-\mathrm{Cl1}$ & $119.2(2)$ \\
\hline $\mathrm{F} 1-\mathrm{C} 15-\mathrm{C} 16$ & $110.4(15)$ \\
\hline $\mathrm{F} 1-\mathrm{C} 15-\mathrm{C} 14$ & $127.9(16)$ \\
\hline $\mathrm{C} 16-\mathrm{C} 15-\mathrm{C} 14$ & $121.6(2)$ \\
\hline $\mathrm{C} 15-\mathrm{C} 16-\mathrm{C} 17$ & $119.50(19)$ \\
\hline
\end{tabular}

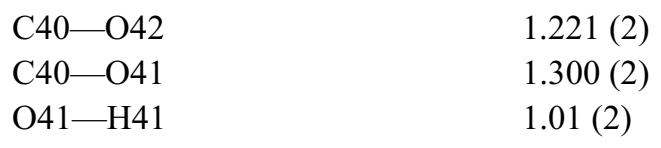


$\mathrm{C} 15-\mathrm{C} 16-\mathrm{H} 16$

$\mathrm{C} 17-\mathrm{C} 16-\mathrm{H} 16$

$\mathrm{C} 16-\mathrm{C} 17-\mathrm{C} 12$

$\mathrm{C} 16-\mathrm{C} 17-\mathrm{H} 17$

C12-C17-H17

$\mathrm{C} 13^{\prime}-\mathrm{C} 12^{\prime}-\mathrm{C} 17^{\prime}$

$\mathrm{C} 13^{\prime}-\mathrm{C} 12^{\prime}-\mathrm{N} 11$

C17'-C12'-N11

$\mathrm{C} 12^{\prime}-\mathrm{C} 13^{\prime}-\mathrm{C} 14^{\prime}$

C12'- $13^{\prime}-\mathrm{H} 13^{\prime}$

C14'- $13^{\prime}-\mathrm{H}^{\prime} 3^{\prime}$

$\mathrm{C} 13^{\prime}-\mathrm{C} 14^{\prime}-\mathrm{C} 15^{\prime}$

C13'-C14'-C11'

$\mathrm{C} 15^{\prime}-\mathrm{C} 14^{\prime}-\mathrm{C} 11^{\prime}$

$\mathrm{C} 16^{\prime}-\mathrm{C} 15^{\prime}-\mathrm{F} 1^{\prime}$

$\mathrm{C} 16^{\prime}-\mathrm{C} 15^{\prime}-\mathrm{C} 14^{\prime}$

$\mathrm{F} 1^{\prime}-\mathrm{C} 15^{\prime}-\mathrm{C} 14^{\prime}$

$\mathrm{C} 15^{\prime}-\mathrm{C} 16^{\prime}-\mathrm{C} 17^{\prime}$

$\mathrm{C} 15^{\prime}-\mathrm{C} 16^{\prime}-\mathrm{H} 16^{\prime}$

$\mathrm{C} 17^{\prime}-\mathrm{C} 16^{\prime}-\mathrm{H} 16^{\prime}$

$\mathrm{C} 16^{\prime}-\mathrm{C} 17^{\prime}-\mathrm{C} 12^{\prime}$

$\mathrm{C} 16^{\prime}-\mathrm{C} 17^{\prime}-\mathrm{H} 17^{\prime}$

C12'-C17'-H17'

C6-O18-C19

$\mathrm{O} 18-\mathrm{C} 19-\mathrm{C} 20$

O18-C19-H19A

$\mathrm{C} 20-\mathrm{C} 19-\mathrm{H} 19 \mathrm{~A}$

O18-C19-H19B

C20-C19- $\mathrm{H} 19 \mathrm{~B}$

H19A-C19-H19B

$\mathrm{C} 21-\mathrm{C} 20-\mathrm{C} 19$

$\mathrm{C} 21-\mathrm{C} 20-\mathrm{H} 20 \mathrm{~A}$

$\mathrm{C} 19-\mathrm{C} 20-\mathrm{H} 20 \mathrm{~A}$

$\mathrm{C} 21-\mathrm{C} 20-\mathrm{H} 20 \mathrm{~B}$

$\mathrm{C} 19-\mathrm{C} 20-\mathrm{H} 20 \mathrm{~B}$

$\mathrm{H} 20 \mathrm{~A}-\mathrm{C} 20-\mathrm{H} 20 \mathrm{~B}$

$\mathrm{N} 22-\mathrm{C} 21-\mathrm{C} 20$

$\mathrm{N} 22-\mathrm{C} 21-\mathrm{H} 21 \mathrm{~A}$

$\mathrm{C} 20-\mathrm{C} 21-\mathrm{H} 21 \mathrm{~A}$

$\mathrm{N} 22-\mathrm{C} 21-\mathrm{H} 21 \mathrm{~B}$

$\mathrm{C} 20-\mathrm{C} 21-\mathrm{H} 21 \mathrm{~B}$

$\mathrm{H} 21 \mathrm{~A}-\mathrm{C} 21-\mathrm{H} 21 \mathrm{~B}$

$\mathrm{C} 27-\mathrm{N} 22-\mathrm{C} 21$

$\mathrm{C} 27-\mathrm{N} 22-\mathrm{C} 23$

$\mathrm{C} 21-\mathrm{N} 22-\mathrm{C} 23$

$\mathrm{N} 22-\mathrm{C} 23-\mathrm{C} 24$

$\mathrm{N} 22-\mathrm{C} 23-\mathrm{H} 23 \mathrm{~A}$

$\mathrm{C} 24-\mathrm{C} 23-\mathrm{H} 23 \mathrm{~A}$
120.3

120.3

119.7 (2)

120.1

120.1

$116(3)$

89 (2)

$152(3)$

122 (3)

119.2

119.2

$122(3)$

145 (3)

$93(2)$

99 (3)

112 (3)

149 (4)

127 (3)

116.4

116.4

$121(3)$

119.7

119.7

$117.35(12)$

105.66 (12)

110.6

110.6

110.6

110.6

108.7

$113.76(13)$

108.8

108.8

108.8

108.8

107.7

115.03 (15)

108.5

108.5

108.5

108.5

107.5

$112.56(14)$

$108.83(15)$

$107.32(14)$

110.88 (17)

109.5

109.5
C37-C $36-\mathrm{H} 36 \mathrm{~A}$

$\mathrm{C} 35-\mathrm{C} 36-\mathrm{H} 36 \mathrm{~B}$

$\mathrm{C} 37-\mathrm{C} 36-\mathrm{H} 36 \mathrm{~B}$

H36A-C36-H36B

$\mathrm{C} 38-\mathrm{C} 37-\mathrm{C} 36$

C38-C37-H 37 A

C36- C37-H 37 A

C38-C37-H37B

C36-C37-H37B

H37A-C37-H37B

C37-C38-C39

C37-C $38-\mathrm{H} 38 \mathrm{~A}$

C39- $338-\mathrm{H} 38 \mathrm{~A}$

C37- $338-\mathrm{H} 38 \mathrm{~B}$

$\mathrm{C} 39-\mathrm{C} 38-\mathrm{H} 38 \mathrm{~B}$

$\mathrm{H} 38 \mathrm{~A}-\mathrm{C} 38-\mathrm{H} 38 \mathrm{~B}$

$\mathrm{C} 40-\mathrm{C} 39-\mathrm{C} 38$

C $40-\mathrm{C} 39-\mathrm{H} 39 \mathrm{~A}$

C38-C39-H39A

C $40-\mathrm{C} 39-\mathrm{H} 39 \mathrm{~B}$

C38-C39-H39B

H39A-C39-H39B

C34-C $35^{\prime}-\mathrm{C}_{3} 6^{\prime}$

C34-C $35^{\prime}-\mathrm{H} 35 \mathrm{C}$

$\mathrm{C} 36^{\prime}-\mathrm{C} 35^{\prime}-\mathrm{H} 35 \mathrm{C}$

C34-C $35^{\prime}-\mathrm{H} 35 \mathrm{D}$

$\mathrm{C} 36^{\prime}-\mathrm{C} 35^{\prime}-\mathrm{H} 35 \mathrm{D}$

$\mathrm{H} 35 \mathrm{C}-\mathrm{C} 35^{\prime}-\mathrm{H} 35 \mathrm{D}$

$\mathrm{C} 35^{\prime}-\mathrm{C} 36^{\prime}-\mathrm{C} 37^{\prime}$

$\mathrm{C} 35^{\prime}-\mathrm{C} 36^{\prime}-\mathrm{H} 36 \mathrm{C}$

$\mathrm{C} 37^{\prime}-\mathrm{C} 36^{\prime}-\mathrm{H} 36 \mathrm{C}$

$\mathrm{C} 35^{\prime}-\mathrm{C} 36^{\prime}-\mathrm{H} 36 \mathrm{D}$

$\mathrm{C} 37^{\prime}-\mathrm{C} 36^{\prime}-\mathrm{H} 36 \mathrm{D}$

$\mathrm{H} 36 \mathrm{C}-\mathrm{C} 36^{\prime}-\mathrm{H} 36 \mathrm{D}$

C $38^{\prime}-\mathrm{C} 37^{\prime}-\mathrm{C} 36^{\prime}$

$\mathrm{C} 38^{\prime}-\mathrm{C} 37^{\prime}-\mathrm{H} 37 \mathrm{C}$

$\mathrm{C} 36^{\prime}-\mathrm{C} 37^{\prime}-\mathrm{H} 37 \mathrm{C}$

C $38^{\prime}-\mathrm{C} 37^{\prime}-\mathrm{H} 37 \mathrm{D}$

C $36^{\prime}-\mathrm{C} 37^{\prime}-\mathrm{H} 37 \mathrm{D}$

H37C-C $37^{\prime}-\mathrm{H} 37 \mathrm{D}$

$\mathrm{C} 37^{\prime}-\mathrm{C} 38^{\prime}-\mathrm{C} 39^{\prime}$

$\mathrm{C} 37^{\prime}-\mathrm{C} 38^{\prime}-\mathrm{H} 38 \mathrm{C}$

$\mathrm{C} 39^{\prime}-\mathrm{C} 38^{\prime}-\mathrm{H} 38 \mathrm{C}$

$\mathrm{C} 37^{\prime}-\mathrm{C} 38^{\prime}-\mathrm{H} 38 \mathrm{D}$

$\mathrm{C} 39^{\prime}-\mathrm{C} 38^{\prime}-\mathrm{H} 38 \mathrm{D}$

$\mathrm{H} 38 \mathrm{C}-\mathrm{C} 38^{\prime}-\mathrm{H} 38 \mathrm{D}$

$\mathrm{C} 40-\mathrm{C} 39^{\prime}-\mathrm{C} 38^{\prime}$

$\mathrm{C} 40-\mathrm{C} 39^{\prime}-\mathrm{H} 39 \mathrm{C}$
109.2

109.2

109.2

107.9

113.7 (2)

108.8

108.8

108.8

108.8

107.7

114.0 (2)

108.7

108.7

108.7

108.7

107.6

111.9 (2)

109.2

109.2

109.2

109.2

107.9

$117.2(8)$

108.0

108.0

108.0

108.0

107.2

113.9 (8)

108.8

108.8

108.8

108.8

107.7

114.0 (7)

108.8

108.8

108.8

108.8

107.6

115.5 (8)

108.4

108.4

108.4

108.4

107.5

119.4 (8)

107.5 


\begin{tabular}{|c|c|c|c|}
\hline $\mathrm{N} 22-\mathrm{C} 23-\mathrm{H} 23 \mathrm{~B}$ & 109.5 & $\mathrm{C} 38^{\prime}-\mathrm{C} 39^{\prime}-\mathrm{H} 39 \mathrm{C}$ & 107.5 \\
\hline $\mathrm{C} 24-\mathrm{C} 23-\mathrm{H} 23 \mathrm{~B}$ & 109.5 & $\mathrm{C} 40-\mathrm{C} 39^{\prime}-\mathrm{H} 39 \mathrm{D}$ & 107.5 \\
\hline $\mathrm{H} 23 \mathrm{~A}-\mathrm{C} 23-\mathrm{H} 23 \mathrm{~B}$ & 108.1 & $\mathrm{C} 38^{\prime}-\mathrm{C} 39^{\prime}-\mathrm{H} 39 \mathrm{D}$ & 107.5 \\
\hline $\mathrm{O} 25-\mathrm{C} 24-\mathrm{C} 23$ & $111.37(19)$ & $\mathrm{H} 39 \mathrm{C}-\mathrm{C} 39^{\prime}-\mathrm{H} 39 \mathrm{D}$ & 107.0 \\
\hline $\mathrm{O} 25-\mathrm{C} 24-\mathrm{H} 24 \mathrm{~A}$ & 109.4 & $\mathrm{O} 42-\mathrm{C} 40-\mathrm{O} 41$ & $124.03(16)$ \\
\hline $\mathrm{C} 23-\mathrm{C} 24-\mathrm{H} 24 \mathrm{~A}$ & 109.4 & $\mathrm{O} 42-\mathrm{C} 40-\mathrm{C} 39^{\prime}$ & $129.0(6)$ \\
\hline $\mathrm{O} 25-\mathrm{C} 24-\mathrm{H} 24 \mathrm{~B}$ & 109.4 & $\mathrm{O} 41-\mathrm{C} 40-\mathrm{C} 39^{\prime}$ & $104.8(5)$ \\
\hline $\mathrm{C} 23-\mathrm{C} 24-\mathrm{H} 24 \mathrm{~B}$ & 109.4 & $\mathrm{O} 42-\mathrm{C} 40-\mathrm{C} 39$ & $118.8(2)$ \\
\hline $\mathrm{H} 24 \mathrm{~A}-\mathrm{C} 24-\mathrm{H} 24 \mathrm{~B}$ & 108.0 & $\mathrm{O} 41-\mathrm{C} 40-\mathrm{C} 39$ & $117.10(19)$ \\
\hline $\mathrm{C} 24-\mathrm{O} 25-\mathrm{C} 26$ & $108.91(17)$ & $\mathrm{C} 40-\mathrm{O} 41-\mathrm{H} 41$ & 109.5 \\
\hline $\mathrm{C} 9-\mathrm{N} 1-\mathrm{C} 2-\mathrm{N} 3$ & $-0.2(3)$ & $\mathrm{N} 11-\mathrm{C} 12^{\prime}-\mathrm{C} 13^{\prime}-\mathrm{C} 14^{\prime}$ & $171.7(16)$ \\
\hline $\mathrm{N} 1-\mathrm{C} 2-\mathrm{N} 3-\mathrm{C} 4$ & $-0.1(3)$ & $\mathrm{C} 12^{\prime}-\mathrm{C} 13^{\prime}-\mathrm{C} 14^{\prime}-\mathrm{C} 15^{\prime}$ & $1(2)$ \\
\hline $\mathrm{C} 2-\mathrm{N} 3-\mathrm{C} 4-\mathrm{N} 11$ & $-178.27(15)$ & $\mathrm{C} 12^{\prime}-\mathrm{C} 13^{\prime}-\mathrm{C} 14^{\prime}-\mathrm{C} 11^{\prime}$ & $-168(3)$ \\
\hline $\mathrm{C} 2-\mathrm{N} 3-\mathrm{C} 4-\mathrm{C} 10$ & $1.1(2)$ & $\mathrm{C} 13^{\prime}-\mathrm{C} 14^{\prime}-\mathrm{C} 15^{\prime}-\mathrm{C} 16^{\prime}$ & $-2(5)$ \\
\hline $\mathrm{C} 10-\mathrm{C} 5-\mathrm{C} 6-\mathrm{O} 18$ & $-179.65(15)$ & $\mathrm{C} 11^{\prime}-\mathrm{C} 14^{\prime}-\mathrm{C} 15^{\prime}-\mathrm{C} 16^{\prime}$ & $171(4)$ \\
\hline $\mathrm{C} 10-\mathrm{C} 5-\mathrm{C} 6-\mathrm{C} 7$ & $-0.4(2)$ & $\mathrm{C} 13^{\prime}-\mathrm{C} 14^{\prime}-\mathrm{C} 15^{\prime}-\mathrm{F} 1^{\prime}$ & $177(6)$ \\
\hline $\mathrm{O} 18-\mathrm{C} 6-\mathrm{C} 7-\mathrm{O} 28$ & $0.7(2)$ & $\mathrm{C} 11^{\prime}-\mathrm{C} 14^{\prime}-\mathrm{C} 15^{\prime}-\mathrm{F} 1^{\prime}$ & $-10(7)$ \\
\hline $\mathrm{C} 5-\mathrm{C} 6-\mathrm{C} 7-\mathrm{O} 28$ & $-178.59(15)$ & $\mathrm{F} 1^{\prime}-\mathrm{C} 15^{\prime}-\mathrm{C} 16^{\prime}-\mathrm{C} 17^{\prime}$ & $177(3)$ \\
\hline $\mathrm{O} 18-\mathrm{C} 6-\mathrm{C} 7-\mathrm{C} 8$ & $-179.53(15)$ & $\mathrm{C} 14^{\prime}-\mathrm{C} 15^{\prime}-\mathrm{C} 16^{\prime}-\mathrm{C} 17^{\prime}$ & $-4(6)$ \\
\hline $\mathrm{C} 5-\mathrm{C} 6-\mathrm{C} 7-\mathrm{C} 8$ & $1.2(3)$ & $\mathrm{C} 15^{\prime}-\mathrm{C} 16^{\prime}-\mathrm{C} 17^{\prime}-\mathrm{C} 12^{\prime}$ & $11(6)$ \\
\hline $\mathrm{O} 28-\mathrm{C} 7-\mathrm{C} 8-\mathrm{C} 9$ & $179.02(15)$ & $\mathrm{C} 13^{\prime}-\mathrm{C} 12^{\prime}-\mathrm{C} 17^{\prime}-\mathrm{C} 16^{\prime}$ & $-11(4)$ \\
\hline $\mathrm{C} 6-\mathrm{C} 7-\mathrm{C} 8-\mathrm{C} 9$ & $-0.7(2)$ & $\mathrm{N} 11-\mathrm{C} 12^{\prime}-\mathrm{C} 17^{\prime}-\mathrm{C} 16^{\prime}$ & $-161(4)$ \\
\hline $\mathrm{C} 2-\mathrm{N} 1-\mathrm{C} 9-\mathrm{C} 10$ & $-0.5(2)$ & $\mathrm{C} 5-\mathrm{C} 6-\mathrm{O} 18-\mathrm{C} 19$ & $-1.1(2)$ \\
\hline $\mathrm{C} 2-\mathrm{N} 1-\mathrm{C} 9-\mathrm{C} 8$ & $179.41(16)$ & $\mathrm{C} 7-\mathrm{C} 6-\mathrm{O} 18-\mathrm{C} 19$ & $179.61(14)$ \\
\hline $\mathrm{C} 7-\mathrm{C} 8-\mathrm{C} 9-\mathrm{N} 1$ & $179.62(15)$ & $\mathrm{C} 6-\mathrm{O} 18-\mathrm{C} 19-\mathrm{C} 20$ & $174.33(14)$ \\
\hline $\mathrm{C} 7-\mathrm{C} 8-\mathrm{C} 9-\mathrm{C} 10$ & $-0.5(2)$ & $\mathrm{O} 18-\mathrm{C} 19-\mathrm{C} 20-\mathrm{C} 21$ & $166.04(15)$ \\
\hline $\mathrm{N} 1-\mathrm{C} 9-\mathrm{C} 10-\mathrm{C} 5$ & $-178.89(15)$ & $\mathrm{C} 19-\mathrm{C} 20-\mathrm{C} 21-\mathrm{N} 22$ & $54.0(2)$ \\
\hline $\mathrm{C} 8-\mathrm{C} 9-\mathrm{C} 10-\mathrm{C} 5$ & $1.2(2)$ & $\mathrm{C} 20-\mathrm{C} 21-\mathrm{N} 22-\mathrm{C} 27$ & $54.86(19)$ \\
\hline $\mathrm{N} 1-\mathrm{C} 9-\mathrm{C} 10-\mathrm{C} 4$ & $1.4(2)$ & $\mathrm{C} 20-\mathrm{C} 21-\mathrm{N} 22-\mathrm{C} 23$ & $174.57(15)$ \\
\hline $\mathrm{C} 8-\mathrm{C} 9-\mathrm{C} 10-\mathrm{C} 4$ & $-178.49(15)$ & $\mathrm{C} 27-\mathrm{N} 22-\mathrm{C} 23-\mathrm{C} 24$ & $-54.7(2)$ \\
\hline $\mathrm{C} 6-\mathrm{C} 5-\mathrm{C} 10-\mathrm{C} 9$ & $-0.7(2)$ & $\mathrm{C} 21-\mathrm{N} 22-\mathrm{C} 23-\mathrm{C} 24$ & $-176.76(17)$ \\
\hline $\mathrm{C} 6-\mathrm{C} 5-\mathrm{C} 10-\mathrm{C} 4$ & $178.92(16)$ & $\mathrm{N} 22-\mathrm{C} 23-\mathrm{C} 24-\mathrm{O} 25$ & $57.9(2)$ \\
\hline $\mathrm{N} 3-\mathrm{C} 4-\mathrm{C} 10-\mathrm{C} 9$ & $-1.7(2)$ & $\mathrm{C} 23-\mathrm{C} 24-\mathrm{O} 25-\mathrm{C} 26$ & $-59.2(2)$ \\
\hline $\mathrm{N} 11-\mathrm{C} 4-\mathrm{C} 10-\mathrm{C} 9$ & $177.61(15)$ & $\mathrm{C} 24-\mathrm{O} 25-\mathrm{C} 26-\mathrm{C} 27$ & $60.6(2)$ \\
\hline $\mathrm{N} 3-\mathrm{C} 4-\mathrm{C} 10-\mathrm{C} 5$ & $178.60(16)$ & $\mathrm{C} 21-\mathrm{N} 22-\mathrm{C} 27-\mathrm{C} 26$ & $173.58(15)$ \\
\hline $\mathrm{N} 11-\mathrm{C} 4-\mathrm{C} 10-\mathrm{C} 5$ & $-2.1(3)$ & $\mathrm{C} 23-\mathrm{N} 22-\mathrm{C} 27-\mathrm{C} 26$ & $54.74(19)$ \\
\hline $\mathrm{N} 3-\mathrm{C} 4-\mathrm{N} 11-\mathrm{C} 12$ & $-0.6(3)$ & $\mathrm{O} 25-\mathrm{C} 26-\mathrm{C} 27-\mathrm{N} 22$ & $-59.3(2)$ \\
\hline $\mathrm{C} 10-\mathrm{C} 4-\mathrm{N} 11-\mathrm{C} 12$ & $-179.94(18)$ & $\mathrm{C} 8-\mathrm{C} 7-\mathrm{O} 28-\mathrm{C} 29$ & $-3.9(2)$ \\
\hline $\mathrm{N} 3-\mathrm{C} 4-\mathrm{N} 11-\mathrm{C} 12^{\prime}$ & $-3.5(12)$ & $\mathrm{C} 6-\mathrm{C} 7-\mathrm{O} 28-\mathrm{C} 29$ & $175.88(15)$ \\
\hline $\mathrm{C} 10-\mathrm{C} 4-\mathrm{N} 11-\mathrm{C} 12^{\prime}$ & $177.1(11)$ & $\mathrm{O} 31-\mathrm{C} 32-\mathrm{C} 33-\mathrm{C} 34$ & $41.3(3)$ \\
\hline $\mathrm{C} 4-\mathrm{N} 11-\mathrm{C} 12-\mathrm{C} 17$ & $-29.1(3)$ & $\mathrm{O} 30-\mathrm{C} 32-\mathrm{C} 33-\mathrm{C} 34$ & $-140.79(17)$ \\
\hline $\mathrm{C} 4-\mathrm{N} 11-\mathrm{C} 12-\mathrm{C} 13$ & $153.23(19)$ & $\mathrm{C} 32-\mathrm{C} 33-\mathrm{C} 34-\mathrm{C} 35^{\prime}$ & $-160.0(6)$ \\
\hline $\mathrm{C} 17-\mathrm{C} 12-\mathrm{C} 13-\mathrm{C} 14$ & $-1.3(3)$ & $\mathrm{C} 32-\mathrm{C} 33-\mathrm{C} 34-\mathrm{C} 35$ & $-174.15(19)$ \\
\hline $\mathrm{N} 11-\mathrm{C} 12-\mathrm{C} 13-\mathrm{C} 14$ & $176.4(2)$ & $\mathrm{C} 33-\mathrm{C} 34-\mathrm{C} 35-\mathrm{C} 36$ & $179.3(2)$ \\
\hline $\mathrm{C} 12-\mathrm{C} 13-\mathrm{C} 14-\mathrm{C} 15$ & $0.1(3)$ & $\mathrm{C} 34-\mathrm{C} 35-\mathrm{C} 36-\mathrm{C} 37$ & $179.9(2)$ \\
\hline $\mathrm{C} 12-\mathrm{C} 13-\mathrm{C} 14-\mathrm{Cl1}$ & $178.38(17)$ & $\mathrm{C} 35-\mathrm{C} 36-\mathrm{C} 37-\mathrm{C} 38$ & $-178.5(2)$ \\
\hline $\mathrm{C} 13-\mathrm{C} 14-\mathrm{C} 15-\mathrm{F} 1$ & $-177(2)$ & $\mathrm{C} 36-\mathrm{C} 37-\mathrm{C} 38-\mathrm{C} 39$ & $-177.9(2)$ \\
\hline
\end{tabular}




$\begin{array}{llll}\mathrm{C} 11-\mathrm{C} 14-\mathrm{C} 15-\mathrm{F} 1 & 5(2) & \mathrm{C} 37-\mathrm{C} 38-\mathrm{C} 39-\mathrm{C} 40 & -69.0(3) \\ \mathrm{C} 13-\mathrm{C} 14-\mathrm{C} 15-\mathrm{C} 16 & 1.2(4) & \mathrm{C} 33-\mathrm{C} 34-\mathrm{C} 35^{\prime}-\mathrm{C} 36^{\prime} & 169.2(6) \\ \mathrm{C} 11-\mathrm{C} 14-\mathrm{C} 15-\mathrm{C} 16 & -177.0(2) & \mathrm{C} 34-\mathrm{C} 35^{\prime}-\mathrm{C} 36^{\prime}-\mathrm{C} 37^{\prime} & 179.8(8) \\ \mathrm{F} 1-\mathrm{C} 15-\mathrm{C} 16-\mathrm{C} 17 & 176.9(19) & \mathrm{C} 35^{\prime}-\mathrm{C} 36^{\prime}-\mathrm{C} 37^{\prime}-\mathrm{C} 38^{\prime} & -67.5(11) \\ \mathrm{C} 14-\mathrm{C} 15-\mathrm{C} 16-\mathrm{C} 17 & -1.3(4) & \mathrm{C}^{\prime} 6^{\prime}-\mathrm{C} 37^{\prime}-\mathrm{C} 38^{\prime}-\mathrm{C} 39^{\prime} & -169.1(9) \\ \mathrm{C} 15-\mathrm{C} 16-\mathrm{C} 17-\mathrm{C} 12 & 0.1(4) & \mathrm{C} 37^{\prime}-\mathrm{C} 38^{\prime}-\mathrm{C} 39^{\prime}-\mathrm{C} 40 & -179.1(9) \\ \mathrm{C} 13-\mathrm{C} 12-\mathrm{C} 17-\mathrm{C} 16 & 1.2(3) & \mathrm{C} 38^{\prime}-\mathrm{C} 39^{\prime}-\mathrm{C} 40-\mathrm{O} 42 & -103.4(10) \\ \mathrm{N} 11-\mathrm{C} 12-\mathrm{C} 17-\mathrm{C} 16 & -176.4(2) & \mathrm{C} 38^{\prime}-\mathrm{C} 39^{\prime}-\mathrm{C} 40-\mathrm{O} 41 & 60.0(11) \\ \mathrm{C} 4-\mathrm{N} 11-\mathrm{C} 12^{\prime}-\mathrm{C} 13^{\prime} & 158.5(10) & \mathrm{C} 38-\mathrm{C} 39-\mathrm{C} 40-\mathrm{O} 42 & -99.5(3) \\ \mathrm{C} 4-\mathrm{N} 11-\mathrm{C} 12^{\prime}-\mathrm{C} 17^{\prime} & -49(5) & \mathrm{C} 38-\mathrm{C} 39-\mathrm{C} 40-\mathrm{O} 41 & 83.0(3) \\ \mathrm{C} 17^{\prime}-\mathrm{C} 12^{\prime}-\mathrm{C} 13^{\prime}-\mathrm{C} 14^{\prime} & 5(2) & & \end{array}$

Hydrogen-bond geometry $\left(\AA,{ }^{\circ}\right)$

\begin{tabular}{|c|c|c|c|c|}
\hline$D-\mathrm{H} \cdots A$ & $D-\mathrm{H}$ & $\mathrm{H} \cdots A$ & $D \cdots A$ & $D-\mathrm{H} \cdots A$ \\
\hline $\mathrm{N} 11-\mathrm{H} 11 \cdots \mathrm{O} 42^{\mathrm{i}}$ & $0.84(2)$ & $2.20(2)$ & $3.025(2)$ & $169.6(18)$ \\
\hline $\mathrm{O} 41-\mathrm{H} 41 \cdots \mathrm{N} 22^{\mathrm{ii}}$ & 1.01 & 1.78 & $2.6566(19)$ & 144 \\
\hline $\mathrm{O} 30-\mathrm{H} 30 \cdots \mathrm{N} 1$ & $0.99(3)$ & $1.65(3)$ & 2.6135 (19) & $166(2)$ \\
\hline $\mathrm{C} 5-\mathrm{H} 5 \cdots \mathrm{O} 42^{\mathrm{i}}$ & 0.95 & 2.25 & $3.194(2)$ & 170 \\
\hline $\mathrm{C} 2-\mathrm{H} 2 \cdots \mathrm{F} 1^{\mathrm{iii}}$ & 0.95 & 2.15 & $3.07(3)$ & 163 \\
\hline $\mathrm{C} 2-\mathrm{H} 2 \cdots \mathrm{F} 1^{\prime \text { iii }}$ & 0.95 & 2.32 & $3.253(3)$ & 166 \\
\hline $\mathrm{C} 29-\mathrm{H} 29 B \cdots \mathrm{O} 18^{\mathrm{iv}}$ & 0.98 & 2.65 & 3.6101 (19) & 167 \\
\hline $\mathrm{C} 23-\mathrm{H} 23 B \cdots \mathrm{O} 25^{\mathrm{v}}$ & 0.99 & 2.57 & $3.220(2)$ & 123 \\
\hline $\mathrm{C} 27-\mathrm{H} 27 B \cdots \mathrm{F} 1^{\mathrm{vi}}$ & 0.99 & 2.71 & $3.68(4)$ & 166 \\
\hline $\mathrm{C} 39-\mathrm{H} 39 A \cdots \mathrm{O} 30^{\mathrm{vii}}$ & 0.99 & 2.50 & $3.255(4)$ & 133 \\
\hline $\mathrm{C} 21-\mathrm{H} 21 B \cdots \mathrm{O} 31^{\text {viii }}$ & 0.99 & 2.29 & $3.234(2)$ & 160 \\
\hline $\mathrm{C} 13-\mathrm{H} 13 \cdots \mathrm{O} 30^{\text {ix }}$ & 0.95 & 2.39 & $3.139(3)$ & 135 \\
\hline $\mathrm{C} 13^{\prime}-\mathrm{H} 13^{\prime} \cdots \mathrm{O} 30^{\mathrm{ix}}$ & 0.95 & 2.53 & $3.268(3)$ & 135 \\
\hline$C g 2 \cdots C g 2^{\text {viii }}$ & & & $3.5358(11)$ & $0(1)$ \\
\hline$C g 2 \cdots C g 3^{\text {viii }}$ & & & $3.7909(11)$ & $1(1)$ \\
\hline$C g 2 \cdots C g 3^{\text {ix }}$ & & & $3.7530(11)$ & $1(1)$ \\
\hline$C g 3 \cdots C g 3^{\text {viii }}$ & & & $3.7934(11)$ & $0(1)$ \\
\hline
\end{tabular}

Symmetry codes: (i) $x-1 / 2,-y+3 / 2, z-1 / 2$; (ii) $x+1 / 2,-y+3 / 2, z+1 / 2$; (iii) $-x,-y+2,-z+1$; (iv) $-x+2,-y+1,-z+1$; (v) $-x+3 / 2, y-1 / 2,-z+1 / 2$; (vi) $x+1, y, z$; (vii) $-x+3 / 2, y+1 / 2,-z+3 / 2$; (viii) $-x+1,-y+1,-z+1$; (ix) $-x+1,-y+2,-z+1$. 